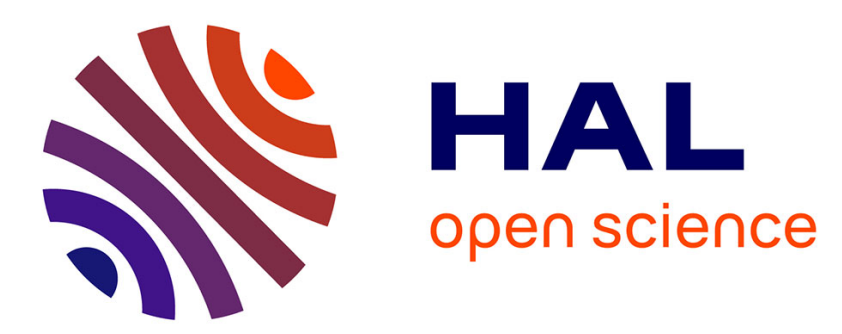

\title{
Novel automated methods for coarse and fine registrations of point clouds in high precision metrology
}

Rindra Rantoson, Hichem Nouira, Nabil Anwer, Charyar Mehdi-Souzani

\section{To cite this version:}

Rindra Rantoson, Hichem Nouira, Nabil Anwer, Charyar Mehdi-Souzani. Novel automated methods for coarse and fine registrations of point clouds in high precision metrology. International Journal of Advanced Manufacturing Technology, 2015, pp.16. 10.1007/s00170-015-7131-1 . hal-01199272

\section{HAL Id: hal-01199272 \\ https://hal.science/hal-01199272}

Submitted on 15 Sep 2015

HAL is a multi-disciplinary open access archive for the deposit and dissemination of scientific research documents, whether they are published or not. The documents may come from teaching and research institutions in France or abroad, or from public or private research centers.
L'archive ouverte pluridisciplinaire HAL, est destinée au dépôt et à la diffusion de documents scientifiques de niveau recherche, publiés ou non, émanant des établissements d'enseignement et de recherche français ou étrangers, des laboratoires publics ou privés. 


\title{
Novel automated methods for coarse and fine registrations of point clouds in high precision metrology
}

\author{
Rindra RANTOSON ${ }^{1}$, Hichem NOUIRA ${ }^{1}$, \\ Nabil ANWER ${ }^{2}$ and Charyar MEHDI-SOUZANI ${ }^{2}$
}

(1): Laboratoire Commun de Métrologie (LNE-CNAM), Laboratoire National de Métrologie et d’Essais (LNE), 1 rue Gaston Boissier, 75015 Paris, France.

(2): Laboratoire Universitaire de Recherche en Production Automatisée (LURPA), Ecole Normale Supérieure de Cachan, Université Paris Sud, 61 avenue du Président Wilson, 94235 Cachan Cedex, France.

*Corresponding authors: hichem.nouira@lne.fr \& $\underline{\text { rindra.sanders@lne.fr }}$

Keywords: Coarse registration, fine registration, Discrete curvatures, Hough Transform, Moving Least Squares Surface, Computational Tomography, Measurement errors, dimensional metrology.

\begin{abstract}
Several measuring systems can be combined to perform accurate assessments at the submicrometre level in dimensional metrology. The obtained data are fused into a common coordinate system using registration methods for which the optimal transformation parameters from the common parts of the data called correspondences are computed. New original automated coarse and fine registration methods are proposed here using discrete curvatures: an improved Hough Transformation method for the coarse registration and three Iterative Closest Points (ICP) variants for the fine registration. The enhancement of Hough consists of exploiting the curvature parameters in order to minimize the basic algorithm complexity. Thus local transformation parameters are only computed for points presenting similar precalculated surface type.

While the rough alignment of the scene data and the model data is thereafter optimized through the fine registration using commonly ICP algorithm, the first ICP variant includes the curvedness and surface type similarity constraints, especially to reduce the searching area during the matching step. For the proposed second ICP variant, correspondences are searched using a specific distance criterion involving curvature features similarity measure defined from principal curvatures. The third ICP variant combines both point-to-point and point-to-plane minimizations automatically
\end{abstract}


weighted in the objective function, with the use of Moving Least Squares (MLS) surface technique to determine the corresponding point in point-to-point part.

The three developed methods are tested on simulated and real data obtained from a computer tomography (CT) system. The results reveal the benefit of the proposed new automated coarse and fine registration approaches.

\section{Introduction}

Measurement of freeform microparts at the sub-micrometre level of accuracy requires one to combine different measuring systems such as computed tomography (CT) and coordinate measuring machines (CMM) based on optical and tactile probing systems. The CT is a technology based on the computer-processed X-rays, which produces tomographic data/image of specific areas of the scanned artefact, allowing the operator to get information about inside and/or outside of the artefact [1-4]. The CMM involves tactile and/or optical measurement only of the outside of the artefact [5-7]. The obtained data are fused into a common coordinate system using coarse and fine registration methods [8]. Registration process aims to compute the optimal transformation parameters $(\boldsymbol{R}, \boldsymbol{T})$ (3 rotation angles in $\boldsymbol{R}$ and 3 translation components in $\boldsymbol{T}$ ) from the common parts of the data called correspondences. An initial rough alignment resulting from the coarse registration is crucial and is thereafter optimized through the fine registration using commonly the ICP algorithm or its variants.

Coarse registration enables to estimate roughly the rigid transformation using commonly marker-based approach [9-11] or automatic shape alignment [12,13]. The second category of methods includes techniques such as the genetic algorithm, and Principal Component Analysis (PCA) [14] which has been robustified recently using the least-median-of-squares method (LMedS) for the principal axis determination in the presence of outliers [15]. This category covers also approaches based on the use of known correspondences found from the calibration relationships between the multiple sensors and views [16] or from similar invariant characteristics, features signatures, etc $[17,18]$.

Fine registration consists of refining the alignment of the two data sets using traditionally ICP algorithm [19]. ICP is an iterative algorithm for the optimal motion estimation that brings the scene data $P$ to the model data $Q$ in closed-form from known correspondences. The iteration is terminated if stopping criteria are reached. At each iteration, ICP is proceeded as follows:

- match points in the overlapping sections of the scene data and the model data $\left(p_{i}, q_{i}\right)$;

- compute the global rigid transformation $\left(\boldsymbol{R}_{\boldsymbol{g}}, \boldsymbol{T}_{\boldsymbol{g}}\right)$;

- apply the motion $\left(\boldsymbol{R}_{\boldsymbol{g}}, \boldsymbol{T}_{\boldsymbol{g}}\right)$ to the scene data points $P$; 
- calculate the error metric;

- apply the stopping criteria.

Different ICP variants are yielded in the literature to improve the algorithm robustness, speed, range and rate of convergence $[20,21]$.

A new automated registration method is proposed in this paper using discrete curvatures. The coarse registration requires no markers and no user interaction since it exploits a curvature feature for an improved HT method [22]. For that purpose, the shape index and the corresponding surface type are computed for all vertices which are afterwards classified accordingly. HT is then applied only on points of the same surface type, which reduces considerably the processing time and the algorithm's complexity.

The exploitation of discrete curvatures has been explored for fine registration while being classified into three approaches.

Although the classical point-to-point ICP algorithm is adopted for the first approach, two additional correspondences matching criteria have been associated with the closest point criterion using respectively the shape index and the curvedness in order to prune the searching area.

For the second approach, a novel distance criterion has been elaborated combining Euclidean distance and curvature distance defined from the principal curvatures. The classical point-to-point minimization algorithm has been applied to compute the transformation parameters.

To improve the robustness of the fine registration to noise, the point-to-point optimization method is combined with a point-to-plane one for the third approach. The curvature parameters of each vertex are exploited for an automatic weights definition and for a local surface approximation of the model data using MLS surface technique ([23]). The final corresponding point taken from the model data is an artificial point defined as the projection of the initial corresponding point onto the local surface approximation.

Finally the developed methods are tested on simulated data and on real data from CT measurements. The results are analysed and reveal the benefits of the automated proposed registration approaches.

\section{Proposed automated registration techniques}

The proposed automated registration process is based on the exploitation of the curvature features of each vertex in scene data $(P)$ and in model data $(Q)$. The shape index, the surface type $\left(T_{s}\right)$ and the local frames $V$ are used for coarse registration while the shape index, the curvedness, the principal curvedness and the surface type are exploited for fine registration. 


\section{1- Curvature extraction}

\section{A. Smooth cases}

For smooth cases, curvatures are defined to measure the local bending of an oriented surface. Assuming a given point $p_{i}$ belongs to the surface, for each unit direction $\vec{u}$ on its tangent plane $T_{p}\left(p_{i}\right)$ the normal curvature $K_{n}(\vec{u})$ is defined as the curvature of the curve that belongs to both the surface itself and a perpendicular plane containing $\vec{n}\left(p_{i}\right)$ and $\vec{u}$. The normal curvature is formulated in Eq.1.

$$
K_{n}(\vec{u})=S_{p_{i}}(\vec{u}) \cdot \vec{u}
$$

Where $S_{p}(\vec{u})$ formulated in (Eq.2) denotes the shape operator (or Weingarten endomorphism) at point $p_{i}$ along the direction $\vec{u}$, which is defined as the derivate of $\vec{n}\left(p_{i}\right)$ with the tangent direction $\vec{u}$, $-\nabla_{\vec{u}} \vec{n}(p)$

$$
S_{p}(\vec{u})=-\nabla_{\vec{u}} \vec{n}(p)
$$

The shape operator matrix is usually symmetric. Its eigenvalues and the corresponding eigenvectors are respectively called the principal curvatures and the principal directions. It has been proved in [24] that the principal curvatures are related with the normal curvature (Eq.3).

$$
K_{n}(\vec{u})=K_{1} \cos ^{2} \theta-K_{2} \sin ^{2} \theta
$$

Where $K_{1}$ and $K_{2}$ represent the maximum and the minimum principal curvatures respectively. $\theta$ is the angle between $\vec{u}$ and the principal direction associated with the maximum principal curvature. These equations allow one to calculate the Gaussian curvature and the mean curvature (the main curvature parameters commonly used) according to the classical definitions and notations of differential geometry for the case of smooth surface [25].

\section{B. Discrete cases}

For discrete models, such as the point cloud or the polygon mesh, the discrete differential geometric information is different from the smooth cases. Classical discrete curvature estimation methods are usually applied on polygon mesh surfaces. Depending on the piecewise smoothness of the input mesh, the discrete curvature estimation is subject to various definitions [26,27]. The classical methods of discrete estimation based on polygon mesh surfaces could be classified into three basic categories: one may approximate a local quadric surface at a given vertex mesh and then apply the derivatives to obtain the curvatures [28]. One may discretize the mathematic formula that gives the curvature information of continuous surface and extend the notations to discrete domains 
[29]. One may use the tensor-based techniques for discrete curvature estimation [28]. The last category has been proved to be efficient and simple to implement, which mainly motivated our choice for a modified Cohen-Steiner [29] approach.

The discrete curvatures on 3D meshes are estimated by the local surface fitting for the first category. For a given vertex $p_{i}$, local parameterization technique, such as local surface fitting, is applied to get a local parametric representation of a surface in $u$ and $v$. Once the $r(u, v)$ corresponds to the semigeodesic coordinates, the Gaussian curvature and mean curvature could be calculated from the following formula (Eq.4).

$$
r(u, v)=(x(u, v), y(u, v), z(u, v))
$$

Although the most commonly used method is the quadric surface approximation, the fitted quadrics are unsuitable for approximating arbitrary data according to Sapidis and Besl [30].

The basic idea of the discrete Laplace-Beltrami operator [31] adopted by Meyer et al [26], which illustrates the second category, is that a mesh is considered as either the limit of a family of smooth surfaces, or a linear approximation of an arbitrary surface. Considering a surface at a vertex $p_{i}$, the geometry properties are defined as the spatial average around this vertex. Thus, it is important to choose the appropriate associated surface patch over which the average will be computed for each vertex. Two main types of local regions are commonly used in practice, but it has been proved by Meyer et al [26] that the Voronoi region presents a better performance than barycentre-based-cell. Similar to the methods based on local quadric surface association, the method of Meyer et al [26] begins with the calculations of mean curvature and Gaussian curvature. The mean curvature normal operator that is also known as Laplace-Beltrami operator is introduced to the discrete case. Since the Gaussian and mean curvatures are both solved, the principal curvatures can be easily computed. To determine the two principal directions, the method uses the tensor based technique and computes the eigenvectors of the curvature tensor. The curvature tensor of a surface $S$ is the map that assigns each point $p_{i}$ of $S$ to the function that measures the normal curvature $K_{n}$ of $S$ at $p_{i}$ in the direction of the unit vector $\bar{u}$, tangent to $S$ at $p_{i}$ [27].

Cohen-Steiner and Morvan proposed a method belonging to the third category that estimates the discrete curvature tensor on polygon mesh [29] by elaborating a curvature measure over a given local region to define the shape operator matrix for each vertex. A variant of their approach has been implemented in our program. 


\section{The adopted dicrete curvature calculation method}

Unlike Cohen-Steiner and Morvan, additional weight coefficient $\lambda_{e}$ has been introduced in the shape operator formulation for the discrete curvature calculation method we propose, in order to consider the contribution of line curvature tensor along each mesh edge to the given vertex. For the local region $B$ definition, Voronoi-cell is generated on the one-ring neighbourhood of the vertex. The shape operator matrix $\boldsymbol{H}$ at each vertex is given by the equation (Eq.5) where $\vec{n}(e)$ is the edge normal at edge $e$, which is equal to the average normal vectors of the two triangles incident to the edge.

$$
\begin{aligned}
& \boldsymbol{H}=\frac{1}{A} \sum_{e \in E} \lambda_{e} \cdot \beta(e) \cdot \text { length }(e \cap B) \cdot(\vec{e} \times \vec{e}) \\
& \lambda_{e}=\frac{\cos ^{-1}(\overrightarrow{\boldsymbol{n}(\boldsymbol{p}),}, \overrightarrow{\boldsymbol{n}(\boldsymbol{e})})}{\sum_{\boldsymbol{e} \in \boldsymbol{E}} \cos ^{-1}(\overrightarrow{\boldsymbol{n}(\boldsymbol{p})}, \overrightarrow{\boldsymbol{n}(\boldsymbol{e}))}}
\end{aligned}
$$

The equation (Eq.5) depends on several parameters such as $\overrightarrow{\mathrm{n}}(\mathrm{e}), \lambda_{\mathrm{e}}, A, e, B$ and $\beta$ illustrated in Fig.1. For any polyhedral mesh surface, the normal to the edge $e, \vec{n}(\mathrm{e})$, is calculated using Eq.7, while the normal vector $\overrightarrow{\mathrm{n}}\left(p_{i}\right)$ at each vertex $p_{i}$ is estimated as the weighted average of the normal vectors of the adjacent triangle facets around it (Eq.8). The unit normal vector $\vec{n}_{i}(i=1 \ldots N)$ of the $i^{\text {th }}$ triangle facet is calculated by Eq.9, which depends on the three unit edge vectors $\vec{e}_{i 1}(j=1,2,3)$ of each $i^{\text {th }}$ triangle facet in counter-clockwise. $\omega_{i}(i=1 \ldots K)$ represents the weight coefficients corresponding to the normal vectors of the facets $f_{i}$. The weight coefficients are defined from the area of each adjacent triangle facet and the distance between the given vertex and the barycenter of each adjacent facet (Eq.10). $A_{i}(i=1 \ldots K)$ represent the area of the $i^{\text {th }}$ triangle facet and $d_{i}(i=1 \ldots N)$ are the distances between the vertex $p_{i}$ and the barycenter of the $i^{\text {th }}$ triangle facet. $N$ is the number of all the triangle facets adjacent to the given vertex.

$$
\begin{aligned}
& \vec{n}(e)=\frac{\vec{n}_{1}+\vec{n}_{2}}{\left\|\vec{n}_{1}+\vec{n}_{2}\right\|} \\
& \vec{n}\left(p_{i}\right)=\frac{\sum_{i=1}^{K} \omega_{i} \cdot \vec{n}_{i}}{\left\|\sum_{i=1}^{N} \omega_{i} \cdot \vec{n}_{i}\right\|} \\
& \vec{n}_{i}=\frac{\vec{e}_{i 1} \times \vec{e}_{i 2}+\vec{e}_{i 2} \times \vec{e}_{i 3}+\vec{e}_{i 3} \times \vec{e}_{i 1}}{\left\|\vec{e}_{i 1} \times \vec{e}_{i 2}+\vec{e}_{i 2} \times \vec{e}_{i 3}+\vec{e}_{i 3} \times \vec{e}_{i 1}\right\|}
\end{aligned}
$$




$$
\omega_{i}=\frac{A_{i} / d_{i}^{2}}{\sum_{i=1}^{K} A_{i} / d_{i}^{2}}
$$

\section{Shape index, curvedness and surface type calculation}

The shape index and the curvedness are two other surface descriptors deduced from the two principal curvatures like the Gaussian and mean curvatures. The shape index first introduced by Koenderink and Doorn [32] is a single value within the range $[-1,1]$ characterizing the local surface type. Regardless of discrete or continuous shapes, the shape index is calculated using Eq.11 where $K_{1}$ and $K_{2}$ represent the maximum and the minimum principal curvatures of the local surface.

$$
\delta=-\frac{2}{\pi} \tan ^{-1}\left(\frac{K_{1}+K_{2}}{K_{1}-K_{2}}\right),\left(K_{1} \geq K_{2}\right)
$$

The surface type is derived from the shape index value as highlighted in Tab.1.

The curvedness $\mathcal{C}$ is a positive number defined as a bending energy specifying the amount of the surface curvature. Its formulation is given in Eq.12 [32]. The curvedness is inversely proportional to the local size of the object and has the dimension of reciprocal length. It only vanishes at planar vertices, while Gaussian curvature vanishes on parabolic surface and mean curvature vanishes on saddle surface.

$$
\mathcal{C}=\sqrt{\frac{K_{1}^{2}+K_{2}^{2}}{2}}
$$

\section{2- Coarse registration}

The previous works on coarse registration are generally achieved manually from known correspondences. Since the correspondences are unknown, the existing automatic methods mostly suffer from the problems of larger matching errors [12] or complex computations [33]. Therefore, different methods are suggested in literature to roughly align the data sets thus avoiding the matching step. Among them, PCA is the most common approach used when the overlapping sections are almost complete and the objects present no symmetries.

In this paper, Hough transformation method has been adopted to automate the rough alignment of two data sets (scene data $P$ and model data $Q$ ) while coping with PCA limitations. An initial HT approach was attempted by Merlin and Farber [34] where only translations of the target object were considered. More recently, Ballard [35] extended the HT approach by including rotations and scale changes for the detection of non-parametric curves. These publications point out the algorithm 
complexity which increases with the data volume since HT is based on an exhaustive search. Hence, the combination of the curvature parameters in the HT principle is aimed at reducing the number of the exhaustive search operations which consequently decreases the computational time as well as the memory storage necessary for the Hough table.

\section{A. Curvature-based Hough Transformation method}

First, local frames defined by the Eigen vectors $\left(V_{i}, V_{j}\right)$ related to the Eigen value decomposition of the shape operator matrix $\boldsymbol{H}$ are calculated for all vertices in both data sets (respectively in scene data $P$ and in model data $Q$ ).

The precalculated local frames $\left(V_{i}, V_{j}\right)$ are afterwards employed to compute the possible global transformation parameters $\left(\boldsymbol{R}_{\boldsymbol{i} \rightarrow \boldsymbol{j}}, \boldsymbol{T}_{\boldsymbol{i} \rightarrow \boldsymbol{j}}\right)$ for all combinations between each point $p_{\boldsymbol{j}}$ in $P$ and each point $q_{i}$ in $Q$ using Eq.13.

$$
R_{i \rightarrow j}=V_{j} . V_{i}^{T} \text { and } T_{i \rightarrow j}=p_{j}-R_{i \rightarrow j} p_{i}
$$

Each new transformation parameters are stored in the Hough table (also called 6D Hough Counting Space (HCS) or the 6D accumulation table) while initializing the corresponding counter to one, which will be incremented according to the number of the transformation parameters occurrences. The correct point correspondences in the scene data and in the model data results in rather identical transformation parameters $\left(\boldsymbol{R}_{\boldsymbol{c}}, \boldsymbol{T}_{\boldsymbol{c}}\right)$, when the residual $\varepsilon_{c}$ in (Eq.14) is less than $10^{-2}$, while the other transformation parameters are distributed randomly in the HCS.

$$
\operatorname{dist}\left(\left(\boldsymbol{R}_{\mathrm{i} \rightarrow \mathrm{j}}, \boldsymbol{T}_{\mathrm{i} \rightarrow \mathrm{j}}\right)-(\boldsymbol{R}, \boldsymbol{T})_{\mathrm{HCS}}\right)<\varepsilon_{c}
$$

The retained transformation is selected regarding the counter values. The counter associated with the searched transformation is expected to provide the highest number of votes in HCS. Furthermore, the peak value would be equal to the number of points correspondences in the overlapping surface when the model data and the scene data match perfectly. The application of the HT requests one to calculate the local transformation parameters between each point in the model data and each point in the scene data, which presents $o(N \mathrm{xM})$ complexity if $N$ and $\mathrm{M}$ denotes respectively the number of points in $P$ and in $Q$ (Fig.2). Hence, the HT approach is a very timeconsuming algorithm for two large cloud data. To solve this problem, we propose to reduce the number of operations by considering curvature parameters precalculated at each point in HT process. Therefore, transformation operations (Eq.13) are only executed between points presenting the same surface type (which is of the number of 10 (Tab.1)). The complexity becomes 
$o\left(\sum_{i=1}^{10}\left(N_{i}^{\prime} \times M_{i}^{\prime}\right)\right)$ where $N_{i}^{\prime}$ and $M_{i}^{\prime}$ are the number of points presenting identical surface type $i$ in $P$ and in $Q$ (Fig.3).

E.g.: 45 local transformation parameters $\left(\boldsymbol{R}_{\boldsymbol{i} \rightarrow \boldsymbol{j}}, \boldsymbol{T}_{\boldsymbol{i} \rightarrow \boldsymbol{j}}\right)$ are calculated for the selected 3 points with the blue colour in the model data covering all possible combinations in Fig.3, while only 21 local transformation parameters $\left(\boldsymbol{R}_{\boldsymbol{i} \rightarrow \boldsymbol{j}}, \boldsymbol{T}_{\boldsymbol{i} \rightarrow \boldsymbol{j}}\right)$ are calculated for the same selected 3 points with blue colour in the model data point when considering the curvature parameters in Fig.3. For both cases local transformation parameters $\left(\boldsymbol{R}_{\boldsymbol{i} \rightarrow \boldsymbol{j}}, \boldsymbol{T}_{\boldsymbol{i} \rightarrow \boldsymbol{j}}\right)$ corresponding to the blue peak are selected for the coarse alignment.

\section{3- Fine registration}

The classical ICP or its variant used for fine registration is mainly operated in two separate and successive steps: the correspondences searching and the optimization. The searching of point pairs in the overlap area of the two discrete shapes constitute an important part of the registration process impacting the quality of the final result. The closest point criterion according to Euclidean distance is traditionally used for the correspondences searching, while point-to-point minimization algorithm or point-to-plane minimization algorithm is generally implemented to find the optimal transformation parameters (the rotation matrix $\boldsymbol{R}_{\boldsymbol{g}}$ and the translation vector $\boldsymbol{T}_{\boldsymbol{g}}$ ). The introduction of the curvature information in the two main steps of the fine registration process has been investigated into three approaches.

\section{A. Method 1: ICP variant using shape index and curvedness for correspondences searching}

Since the curvature information is geometry invariant, the points corresponding to the same point on a physical object should have similar curvature attributes. The similarity between two points can be defined based on second order geometric attributes such as the shape index (surface type) and the curvedness. During the matching step, three criteria related to the closest point position and curvature parameters similarity have been implemented to perform ICP algorithm. For a given point $p_{i}$ in scene data $P$, its corresponding point in model data $Q$ is the closest point $q_{i}$ presenting the same surface type (shape index) and the same curvature as $p_{i}$, equation (14 bis). The feature distance $d_{f}$ defined in Eq.15 is used to measure the curvature parameters similarity between two points, $p_{i}$ in $P$ and $q_{i}$ in $Q$.

$$
d_{f}=\left\{\begin{array}{l}
\mathcal{C}_{p_{i}}-\mathcal{C}_{q_{i}} \text { if } T_{s}\left(p_{i}\right)=T_{s}\left(q_{i}\right) \\
\infty \quad \text { else } T_{s}\left(p_{i}\right) \neq T_{s}\left(q_{i}\right)
\end{array}\right.
$$




$$
R_{p q}=\underset{q_{i} \in \psi\left(p_{i}\right)}{\operatorname{argmin}} \operatorname{dist}\left(p_{i}, q_{i}\right) \text { where } \Psi\left(p_{i}\right)=d_{f}<T h_{d f}
$$

Thus, for any given point $p_{i}$ in $P$, the feature distance $d_{f}$ is first calculated if the corresponding point $q_{i}$ in $Q$ presents the same surface type as $p_{i}$ (Eq.16).

The final corresponding point is then selected among $q_{i}$ points in the subset $\Psi\left(p_{i}\right)$ by applying the closest point criterion within Euclidian distance. $\Psi\left(p_{i}\right)$ is defined using a feature distance threshold $T h_{d f}$, which delimits the matching resolution of two points $p_{i}$ and $q_{i}$ while influencing the accuracy of the correspondences searching results. A large value of $T h_{d f}$ causes the matching resolution to be too large by the algorithm. As a consequence, many point pairs with bad correspondences will be found and considered for the next step of the process. By contrast, a too small value of $T h_{d f}$ leads to discount several good correspondences penalizing the determination of the optimal global transformation parameters $\left(\boldsymbol{R}_{\boldsymbol{g}}, \boldsymbol{T}_{\boldsymbol{g}}\right)$. Both conditions can lead the coarse alignment to failure. In general, if the curvature estimation is reliable, a compromised value of $T h_{d f}$ fixed in the interval of $\left[10^{-3} 10^{-7}\right]$ can induce a satisfying rough alignment.

Once the matched points are stored, the next step consists of computing the global transformation parameters $\left(\boldsymbol{R}_{\boldsymbol{g}}, \boldsymbol{T}_{\boldsymbol{g}}\right)$ by solving the optimization problem in Eq.17 using SVD (Singular Value Decomposition) or UQ (Unit Quaternion) in closed-form. Moreover, Levenberg Marquardt and LBFGS methods were also respectively studied and discussed by NIST and LNE [36] for an optimal solution of the problem (Eq.17).

$$
f\left(R_{g}, T_{g}\right)=\min _{R_{g}, T_{g}} \frac{1}{n} \sum_{i=1}^{n}\left\|R_{g} p_{i}+T_{g}-q_{i}\right\|^{2}
$$

Where $n$ is the number of point pairs.

After numerous iterations, normally less than 10, the overlapping sections between $P$ and $Q$ can be aligned with good performance.

\section{B. Method 2: ICP variant based on curvature distance and Euclidean distance combination}

The accuracy of the found corresponding point pairs affects the estimation of the transformation parameters. The output of this step has a major impact over the downstream stages and influences the overall performance of the registration approach. In order to enhance the performance of this phase, the geometric distance has been introduced for a better recognition of the similarity between points in the overlapping area of $P$ and $Q$. Thus, assuming an arbitrary vertex $p_{i}$ in $P$ and an arbitrary vertex $q_{j}$ in $Q$, the proposed geometric distance $d_{g}$ defined in the equation (Eq.18) 
depends on both Euclidean distance $d_{e}$ (Eq.19) and curvature ratio distance $d_{c}$ (Eq.20) between $p_{i}$ and $q_{j}$.

$$
\operatorname{dist}\left(p_{i}, q_{j}\right)=d_{g}\left(p_{i}, q_{j}\right)=\lambda \cdot d_{e}+(1-\lambda) \cdot k \cdot d_{c}
$$

Where

$$
\begin{aligned}
& d_{e}=\left\|p_{i}-q_{j}\right\| \\
& d_{c}=\sqrt{\left(\rho_{1 p}-\rho_{1 q}\right)^{2}+\left(\rho_{2 p}-\rho_{2 q}\right)^{2}}
\end{aligned}
$$

and $\rho_{1}=1 / K_{1}, \rho_{2}=1 / K_{2}, \lambda \in[0,1]$.

The coefficient $k$ is a constant that normalizes the curvature ratio distance $d_{c}$, while $\lambda$ is a coefficient set by the operator to balance the contribution of $d_{c}$ and $d_{e}$.

Once points are matched using the closest geometric distance criterion, the global transformation parameters $\left(\boldsymbol{R}_{\boldsymbol{g}}, \boldsymbol{T}_{\boldsymbol{g}}\right)$ are computed in the same manner as the previous approach.

A compromising threshold is used to retain or not the found closest point for the sake of reducing the matching errors.

\section{Method 3: ICP variant combining point-to-surface and point-to-plane algorithms}

For the third proposed approach, the research of correspondences can be based on Euclidean distance criterion or on geometric distance criterion previously defined. However, unlike the rigid registration where only known point pairs $\left(p_{i}, q_{i}\right)$, using separately point-to-point or point-to-plane algorithm, are considered, the proposed novel objective function combines both point-to-point and point-to-plane minimization while introducing additional correspondences $\left(p_{i}, q i^{*}\right)$ for the point-topoint part (Eq.21), where $q_{i}^{*}$ is estimated from the initial $q_{i}$ of $\left(p_{i}, q_{i}\right)$ and its neighbours using the MLS surface technique.

$$
f\left(R_{g}, T_{g}, p, q\right)=\sum_{i=1}^{n} \omega_{i}^{*}\left[\alpha_{i}\left\|R p_{i}+T-q_{i}^{*}\right\|^{2}+\beta_{i}\left(\left(R p_{i}+T-q_{i}\right)^{T} n_{i}\right)^{2}\right]
$$

$\omega_{i}^{*}$ are weights close to one indicating the reliable correspondences.

$\left(\alpha_{i}, \beta_{i}\right)$ are automatically set according to the preliminary surface type value estimated for each point (Tab.1). The idea behind this is based on the following assumption: the higher the surface type value is, the higher the value of $\alpha_{i}$ and the lower the value of $\beta_{i}$ are and vice versa (Tab.2). For 
example, if the surface type of a point pair $\left(p_{1}, q_{1}\right)$ is a dome, 0.8 is attributed to $\alpha_{1}$ and 0.2 for $\beta_{1}$. Hence, if the surface type of a point pair $\left(p_{2}, q_{2}\right)$ is a plane, 0.1 is attributed to $\alpha_{2}$ and 0.9 to $\beta_{2}$.

The calculation of the point $q_{i}{ }^{*}$ based on MLS approach is carried out by applying Algebraic Point Set Surfaces (APSS) method [23] (Fig.4). The APSS approximating the point cloud (formed here by the neighbour points of $q_{i}, N\left(q_{i}\right)$ ) yields as the zero set of an implicit scalar function $f(\mathrm{x})$, representing the algebraic distance between the evaluation point $X \in N\left(q_{i}\right)$ and the local fitted sphere $u(X)$, (Eq.22).

$$
f(X)=s_{u}(X)=\left[\begin{array}{lll}
1 & X^{T} & X^{T} X
\end{array}\right] u(X)=0
$$

Where $u=\left[u_{0}, u_{1}, u_{2}, u_{3}, u_{4}\right]^{T}$ is the vector of scalar coefficients describing the local sphere $u(X)$ estimated by solving the following optimization problem:

$$
u(X)=\min _{u} \sum_{i} \omega_{i}^{a d}(X)\left(s_{u}\left(q_{i}\right)^{2}+\left\|\nabla s_{u}\left(q_{i}\right)-\vec{n}_{i}\right\|^{2}\right)
$$

Where

$\vec{n}_{i}$ is the surface normal of $q_{i} . \nabla s_{u}\left(q_{i}\right)$ is the gradient of $f(X) . \omega_{i}^{a d}$ is a weight associated to each considered $\mathrm{X}$ formulated in equation (Eq.24).

$$
\omega_{i}^{a d}(X)=\phi\left(\frac{\left\|q_{i}-X\right\|}{r_{i} \cdot h}\right)
$$

Where $\phi$ is a smooth decreasing weight function, $r_{i}$ is the radius representing the local point spacing and $h \in \mathbb{R}$ is a global scale factor adjusting the influence of the radius of every point. $r_{i}$ is computed as the distance from $q_{i}$ to its farthest neighbour point using a neighbourhood definition while $h$ is set intuitively.

Finally the point $q_{i}{ }^{*}$ is found by iteratively projecting the point $q_{i}$ onto the local sphere. The pointto-plane minimization method has been proved to be robust to noise. However, inaccuracies can be induced if the neighbouring points present high curvatures due to the least squares estimation of the tangent plane. The reason is that the new proposed formulation of the objective function includes both the point-to-plane approach to handle points presenting low curvatures and the point-to-point approach to handle points with high curvatures. The proposed ICP variant can be considered as nonrigid registration method and has been proved to be robust to noise.

\section{4- Implementation of the coarse and fine registration algorithm}

The combination of HT method and curvature parameters which guarantees the coarse registration as well as the three ICP variants described above for fine registration, have been implemented using 
MATLAB software on a pc characterized by an Intel core i7/×64 platform with 8 GB of RAM and a $2.0 \mathrm{GHz}$ processor.

The proposed curvature-based registration process which offers three possibilities for fine registration is illustrated in Fig.5 interpretable as follows:

Method 1/ Searching correspondences using Euclidean distance $d_{e}\left(p_{i}, q_{j}\right)$ for points of identical shape index and curvedness followed by the application of the point-to-point minimization method (rigid registration method). This procedure corresponds to the flows $\mathbf{a}$ and $\mathbf{c}$ in Fig.5.

Method 2/ Searching correspondences using specific distance $d_{g}\left(p_{i}, q_{j}\right)$ for all points and application of the point-to-point minimization. This procedure corresponds to the flows $\mathbf{b}$ and $\mathbf{c}$ in Fig.5.

Method 3/ Searching correspondences using specific distance $d_{g}\left(p_{i}, q_{j}\right)$ for all points and application of the point-to-point combined with point-to-plane minimization (non-rigid registration method). This procedure corresponds to the flows $\mathbf{b}$ and $\mathbf{d}$ in Fig.5.

For any selected procedure, the program terminates only if one of the stopping criteria is satisfied: if the difference of the mean squared errors (MSE) between two iterations is weaker than $\varepsilon=10^{-3}$ or if the number of the iterations $N$ is greater than 100 .

\section{Results}

\section{1- Evaluation of the robustness of the developed algorithms on simulated data}

Two simulations were handled in order to study the robustness of the algorithms when confronted to measurements of different levels of noise and when dealing with data fusion. The developed three methods were applied on simulated data collected from the CAD data of a LEGO connector. Initially, more than one million points are generated to define the scene data (the moving set). The corresponding curvature $\mathcal{C}$ and shape index $\delta$ parameters are illustrated respectively in Fig.6(a) and (b). The model data (the fixed set) is thereafter built by combining a generated Gaussian noise to a copy of the CAD data. For the first simulation, three tests involve generating Gaussian noise with controlled mean and standard deviation $(\mu=0, \sigma=5,50$ and $100 \mathrm{~nm})$. The considered values of the standard deviation correspond to typical noise values observed on the measuring system. $\sigma=5 \mathrm{~nm}$ corresponds to the noise caused by the probing system integrated on ultra-high precision CMM [5,6], while $\sigma=50 \mathrm{~nm}$ corresponds to the noise that can be seen on classical CMM. $\sigma>100 \mathrm{~nm}$ corresponds to the noise produced by the measuring system integrated on $\mu \mathrm{CT}$. 
The Matlab function "randn" is used to generate the Gaussian noise (Fig.7), added in the orthogonal direction at each model data point. The execution of this function returns actual standard deviation that slightly differs from the imposed value. For the analysis, RMS and PV values calculated from the generated Gaussian noise are consequently considered as the true generated values. Once the model data is created, it is translated $\left(T_{x}=2 \mathrm{~mm}, T_{y}=-3\right.$ and $\left.T_{z}=-1 \mathrm{~mm}\right)$ and rotated $\left(R_{x}=-0.5 \mathrm{rad}, R_{y}=-0.01 \mathrm{rad}\right.$ and $\left.R_{\mathrm{z}}=0.5 \mathrm{rad}\right)$ along $\mathrm{x}-, \mathrm{y}$ - and z-axis to generate the initial alignment between the two data sets, Fig.8(a).

Afterwards, the three developed registration methods, which have in common the enhanced HT registration method, are applied. The coarse and the fine registration results obtained using the first approach, are respectively illustrated in Fig.8(b) and in Fig.8(c).

Finally, the RMS and PV of the residual registration errors are evaluated and compared to the RMS and PV of the generated noise since they transcribe the form specifications of the LEGO connector. For the first method, the influence of $T h_{d f}$ (Eq.15) and the Euclidean distance threshold $T h_{\text {dist }}$ (to delimit the accepted closest points distance) on MSE are evaluated for a noise level of $\sigma=5 \mathrm{~nm}$. For such a level of noise the algorithm converges only if $T h_{\text {dist }}$ varies between 0 and 0.05 and if $T h_{d f}$ varies between 0 and 0.1 . The results illustrated in Fig.9, considered as an abacus, reveal that the MSE values are low for $T h_{d i s t}$ less than $0.01 \mathrm{~mm}$ and for $T h_{d f}$ varying between 0.001 and 0.005 range. For a noise level of $\sigma=50 \mathrm{~nm}$, the algorithm converges only if $T h_{\text {dist }}$ varies between 0 and 0.5 and if $T h_{d f}$ varies between 0 and 0.1 . The MSE values are low when $T h_{d i s t}$ is less than $0.2 \mathrm{~mm}$ or when $T h_{d f}$ varies between 0.0001 and 0.003 range.

For the second and third methods (for which we recall that the parameters $\alpha_{i}$ and $\beta_{i}$ are set automatically in the program according to the shape index parameter as indicated in Tab.2), the influence of the parameter $\lambda\left(\lambda \in\left[\begin{array}{ll}0 & 1\end{array}\right]\right)$ (Eq.6) on MSE values is investigated with the three proposed levels of noise. The obtained MSE using Euclidean distance $(\lambda=1)$ is more satisfying compared with the MSE values obtained by varying $\lambda$.

Focussing on results obtained using the methods (1) and (3), the difference between the generated and the estimated RMS and PV, respectively denoted $\Delta(P V)$ and $\Delta(R M S)$, is small within low MSE values too (Tab.3). Additionally, the MSE values issued of the first method increase from $\sim 10^{-31}$ to $\sim 10^{-11}$ when the amplitude of noise increases. Regarding the third method, MSE values increase slowly from $\sim 10^{-11}$ to $\sim 10^{-9}$.

For the data fusion simulation, the model data is combined with the Gaussian noise of $\sigma=$ $5 \mathrm{~nm}$ and the scene data is combined with the Gaussian noise of $\sigma=100 \mathrm{~nm}$. The results reported in Tab.4 confirm that the methods (1) and (3) are more accurate than the proposed method (2). The 
deviations $\Delta(P V)$ and $\Delta(R M S)$ related to the methods (1) and (3) are below a nanometre. The test is repeated again for which the model data is combined with Gaussian noise of $\sigma=100 \mathrm{~nm}$ and the scene data is combined with Gaussian noise of $\sigma=5 \mathrm{~nm}$. The results reported in Tab.5 are similar to those presented in Tab.4 and endorse the accuracy of the methods (1) and (3) at a nanometre level.

\section{2- Application of the algorithms on $\mu \mathrm{CT}$ measurement}

The concept of X-ray computer tomography (CT) is based on the attenuation of the X-ray beam through the specimen. According to Beer-Lambert law, the ratio between the transmitted and the incident photons depends on the integral of the absorption coefficient of the material $\mu$ along the path that the photons follow through the specimen. The absorption coefficient $\mu$ is linked to the density, the atomic number and the energy [1-4]. The resulting image is a projection of a volume in a 2D plane. To get 3D information, numerous radiographs should be taken while rotating the specimen between 0 and $180^{\circ}$. These projected images allow one to mathematically reconstruct the volume of the measured specimen using a specific algorithm.

CT requires an X-ray source, a rotation stage and an X-ray detector. Fan, cone and parallel $\mathrm{X}$-ray sources are commonly used. A fluorescent detector is usually used and devoted for the changing of the X-rays into visible light which is transferred to the CCD camera by a set of optic lenses (Fig.10). Both the specimen and the rotary stage can move between the detector and the source using a high precision bearing or air-bearing system in order to adjust the resolution which generally lies between 10 and $500 \mu \mathrm{m}$. The necessary time for a complete scan is strongly influenced by the resolution, the size of the CCD camera and the source therefore is difficult to set a general rule.

Nowadays the term micro-CT ( $\mu \mathrm{CT}$ ) is usually used to refer to tomography with an image spatial resolution in the micron range, which can be achieved if the spot size is within the order of few microns, or via an adequate set of optics in the detector.

The $\mu$ CT used here for the LEGO connector scanning is the Carl Zeiss METROTOM 800. METROTOM 800 was specially achieved through the use of maintenance-free micro-focus X-ray tube technology (Fig.10). Extremely small focal points enable razor-sharp projection images on the detector, leading to the foundation of a high measuring accuracy. The METROTOM 800 detector delivers almost three million pixels for very high detail recognition (Tab.6).

In the METROTOM 800, the specimen can be located at any position along the beam path via a continuously adjustable travel mechanism. The detector is always optimally illuminated with the 
part projection. Together with the vertical adjustment, this function allows one to enlarge specific areas of the parts to measure details in relation to the entire specimen.

The described $\mu \mathrm{CT}$ is used for the scanning of the connector prototype provided from the LEGO Company which is a supporter of the JRPMICR (IND59) project. The connector is made of polycarbonate materials and manufactured by an injection moulding process. The dimensions of the connector are of $25 \mathrm{~mm}$ length and $5 \mathrm{~mm}$ diameter (Fig.11).

More than one million points are recorded to cover the entire specimen surfaces. The registration of the $\mu \mathrm{CT}$ measurement data with the CAD data is carried out using the three implemented methods. For the second and third methods, the impact of the $\lambda$ parameter on MSE is investigated and the results are reported in Fig.12. $\lambda=0.4$ gives the smallest MSE value for both methods (2) and (3) and is considered as the optimal value selected for the fine registration. The obtained results are illustrated in Tab.7. The initial alignment is presented in Fig.13(a), while the coarse and fine registration results are illustrated in Fig.13(b) and (c) respectively. According to the results in Tab.7, the methods (1) and (3) are more accurate than method (2). The average of the three obtained PV values is $87.941 \mu \mathrm{m}$ while the average of the three obtained RMS values is $4.183 \mu \mathrm{m}$.

\section{1- Analysis of the results}

The entire process involving the coarse and the fine registration algorithms are applied and evaluated firstly on simulated data of the LEGO connector CAD and secondly on its measurement performed by the previously described $\mu \mathrm{CT}$ system.

\section{A. Registration of simulated data}

We recall for the first simulation that the model data is defined by the CAD data while three levels of noise ( $\sigma=5,50$ and $100 \mathrm{~nm}$ ) are generated and combined with a copy of the CAD data to create the scene data. The algorithms are then applied to ensure the coarse and fine registrations. Regarding the investigated case, both methods (1) and (3) are more accurate than method (2). The MSE obtained with the method (1) leans towards $10^{-31}$ especially when the generated noise has $\sigma \leq$ $50 \mathrm{~nm}$. The deviation between both generated and estimated PV and RMS is less than 10-15 (Tab.3). It corresponds to the scanning of a freeform standard or a high surface quality of a complex specimen by means of a high precision CMM or an ultra-high precision CMM (accurate at the nanometre level). Thus, in order to guarantee an evaluation at nanometre level, the data registration and its analysis can be performed using method (1) due to its negligible error, which can be assimilated to the software operation inaccuracy. The MSE provided by method (1) is distinctly 
more accurate than the one obtained using the other methods (2) and (3) since it is calculated only on corresponding points of the same curvature parameters (shape index and curvedness) while the MSE related to the two other methods (2) and (3) are calculated without applying this constraint. For method (1), both threshold $T h_{d f}$ and threshold of the Euclidean distance $T h_{\text {dist }}$ should be selected carefully in the abacus (Fig.9), which can be considered as the detriment and weakness of this method (1). Moreover, the three developed methods allow one to analyse a large number of data, more than $1,000,000$ points.

For the simulation with noise amplitude higher than $400 \mathrm{~nm}$, both proposed methods (1) and (3) provide deviations $\Delta(P V)$ and $\Delta(R M S)$ below the nanometre level. The analysis of the deviations $\Delta(P V)$ between the generated and estimated PV reveals again that method (1) is more accurate. Nonetheless, method (2) presents least satisfying results among all presented cases.

Simulations for which model and scene data are both combined with Gaussian noise of different amplitudes (20 nm Gaussian noise amplitude $(\sigma=5 \mathrm{~nm})$ for the model data and $400 \mathrm{~nm}$ Gaussian noise amplitude ( $\sigma=100 \mathrm{~nm}$ ) for the scene data and inversely) are performed in order to investigate the ability of the proposed methods to fuse data provided from different systems of measurement (tactile and optical probing system, from an high precision CMM and an ultra-high precision CMM, from CT system, vision system, etc).

The application of the methods (1), (2) and (3) on simulated data confirms that method (1) is more accurate than the method (3) which both are more accurate than method (2) (Tab.4 and 5). Regarding the amplitude of noise in model and scene data, which is less than $400 \mathrm{~nm}$, the obtained results emphasize once again that the methods (1) and (3) are promising. According to these results, we can consider that the method (1) and (3) are more suitable for dimensional metrology at the nanometre level of uncertainty.

\section{B. Registration of CAD data and measured data from CT tomography}

The second test consists of aligning the CAD data and the measured data using our three implemented algorithms. We recall that the measurement is performed by Carl Zeiss METROTOM $800 \mu \mathrm{CT}$, offering us a large surface dataset covering the entire connector. The obtained measurement is composed of more than 1,000,000 points. The results reported in Tab.7 show that the average PV value is $87.941 \mu \mathrm{m}$ and the average RMS value is $4.183 \mu \mathrm{m}$. The high value of PV can be explained by a combination of the form errors of the connector with the resolution and linearity errors of the $\mu \mathrm{CT}$. These $\mu \mathrm{CT}$ errors generally exceed some tens of micrometres. The form errors of the connector are caused by the manufacturing process based on the injection moulded polycarbonate and by the physical phenomena that appear during cooling. The shape of the 
connector etched in the mould presents form errors related to the error motions of the mechanical guiding stages supporting the movable table of the electrical discharge machining, where the mould is fixed and manufactured. These errors can be considered as systematic errors since they appear in all the manufactured specimen using the same mould. In the other side, the errors generated by $\mu \mathrm{CT}$ can be distinguished into systematic and random errors depending if those are repeatable or not. Numerous errors sources in the $\mu \mathrm{CT}$ can be identified such as: resolution of the source of the X-ray, resolution and linearity errors of the detector, positioning errors of the artefact in the working space, error motions and positioning errors of the spindle, positioning of the detector, reconstruction of the numerous collected 2D radiology, number of orientations of the artefact, etc..

\section{Conclusion}

Three curvature-based registration methods have been reported in this paper to deal with automated alignment of two data sets in dimensional metrology. The classical markers manually identified by the user during the matching step of the coarse registration in industrial applications (such as in reverse-engineering, quality control, etc.) are replaced by the curvature features of the workpiece. These ones are recognized and quantified automatically using a discrete curvature calculation method on meshed data for which a variant of tensor-based techniques has been implemented.

For coarse registration, the three implemented methods are commonly based on an enhanced HT method applied on local regions of similar surface type to improve the basic algorithm while dealing with PCA limitations. Satisfying rough alignments were obtained observing that the result accuracy is curvature quality depending.

For fine registration, curvature parameters were exploited to come up with three variants of ICP methods. The two first approaches adopt point-to-point algorithm for the minimization step, however, the specificity lies in the introduction of curvature features in the matching step. For the first approach, two constraints defined from the shape index and the curvedness are applied during the matching step to prune the correspondences searching area. For the second approach, a new geometric distance merging Euclidean distance with curvature distance defined from principal curvatures, has been elaborated for the correspondences searching.

For the third approach, the objective function of classical ICP algorithm has been substituted by a combination of point-to-point and point-to-plane algorithms with automatic weights determined from the surface type. Moreover, MLS technique has been introduced to find the final corresponding point used in the point-to-point algorithm part. For correspondences searching, 
Euclidean distance or a mixture of this one with a curvature distance can be used to further reduce registration error in some cases.

To evaluate the robustness of the implemented three ICP variants, two simulations were undertaken using the CAD data of a LEGO connector combined with Gaussian noise. The first simulation aims at evaluating the robustness of our algorithms on three measurements issued from a measuring system with three given levels of noise while the second one simulates the data fusion of different levels of noise. The proposed three curvature-based fine registration approaches provide preliminary promising results. The first approach provides the most satisfying registration error in most cases while the second and the third registration errors are more or less convincing depending on the cases. The algorithms have been applied on the LEGO connector measurement performed by Carl Zeiss METROTOM $800 \mu \mathrm{CT}$ with its CAD data. Through the exposed applications the performance induced by curvature parameters introduction for enhanced HT approach has been emphasized. Additionally, the influence of the curvature similarity measure utilized for fine registration as well as the advantage of the novel objective function have been highlighted. Thus, the results revealed the benefit of the new automated proposed registration approaches pointing out the curvature exploitation.

\section{Acknowledgments}

The authors sincerely thank the EMRP organization. The EMRP is jointly funded by the EMRP participating countries within EURAMET and the European Union (JRPMICR: IND59). The authors are grateful to Dr. Brian Hougaard Sørensen from LEGO for providing us the CAD model of the LEGO connector and to Dr. Frank Thibault from Carl Zeiss for the CT measurements on our artefacts. 


\section{References}

[1] Kruth JP, Bartscher M, Carmignato S, Schmitt R, De Chiffre L, Weckenmann A (2011) Computed tomography for dimensional metrology. CIRP Annals - Manufacturing Technology 60: 821-842.

[2] Carmignato S (2012) Accuracy of industrial computed tomography measurements: Experimental results from an international comparison. CIRP Annals - Manufacturing Technology 61: 491-494.

[3] De Chiffre L, Carmignato S, Kruth JP, Schmitt R, Weckenmann A (2014) Industrial applications of computed tomography. CIRP Annals - Manufacturing Technology 63: 655-677.

[4] Salvo L, Cloetens P, Maire E, Zabler S, Blandin JJ, Buffière JY, Ludwig W, Boller E, Bellet D, Josserond C (2003) X-ray micro-tomography an attractive characterisation technique in materials science, Nuclear Instruments and Methods in Physics Research B 200: 273-286.

[5] Nouira H, Bergmans RH, Küng A, Piree H, Henselmans R, Spaan HAM (2014) Ultrahigh precision CMMs and their associated tactile or/and optical scanning probes. International Journal of Metrology and Quality Engineering 5: 13pp.

[6] Claverley JD, Leach RK (2015) A review of the existing performance verification infrastructure for micro-CMMs. Precision Engineering 39: 1-15.

[7] Colosimo BM, Pacella M, Senin N (2015) Multisensor data fusion via Gaussian process models for dimensional and geometric verification. Precision Engineering 40: 199-213.

[8] Franceschini F, Maisano D (2014) The evolution of large-scale dimensional metrology from the perspective of scientific articles and patents. Int J Adv Manuf Technol 70: 887909.

[9] Kim TW, Seo YH, Lee SC, Yang Z, Chang M (2009) Simultaneous registration of multiple views with markers. Computer-Aided Design 41: 231-239.

[10] Rantoson R, Stolz C, Fofi D, Meriaudeau F (2012) Optimization of transparent objects digitization from visible fluorescence UV-induced. Optical Engineering 51: 15 pp (033601).

[11] Zhu L, Barhak J, Srivatsan V, Katz R (2007) Efficient registration for precision inspection of free-form surfaces. Int J Adv Manuf Technol 32: 505-515.

[12] Ameesh M, Alexander PIV, Kostas D (2006) Fully automatic registration of 3D point clouds. Proceedings of IEEE computer society conference on computer vision and pattern recognition (CVPR'06) 2597-2604. 
[13] Shmukler A, Fischer A (2010) Verification of 3D freeform parts by registration of multiscale shape descriptors. Int J Adv Manuf Technol 49: 1093-1106.

[14] Liu B, Ning S, Lin J, Jiang K (2008) Transferring landmarks to individual foot using deformable template model. Proceedings of IEEE $9^{\text {th }}$ International Conference for Young Computer Scientist 677-682.

[15] Liu YS, Ramani K (2009) Robust principal axes determination for point-based shapes using least median of squares. Computer-Aided Design 41: 293-305.

[16] Zheng H, Saupe D, Roth M, Bohler A, Opuchlik P (2008) Efficient 3D shape acquisition and registration using hybrid scanning data. Proceedings of 3DPVT’08: 251258.

[17] Kim K, Woo W (2004) Projection -based registration using color and texture information for virtual environment generation. Springer, PCM, LNCS3331: 434-43.

[18] Li X, Guskov I (2005) Multi-scale features for approximate alignment of point-based surfaces. SGP 217.

[19] Chen Y, Medioni G (1991) Object modeling by registration of multiple range images. Proceedings of IEEE conference on robotics and automation 2724-2729.

[20] Rusinkiewicz S, Levoy M (2001) Efficient variants of the ICP algorithm, Proceedings. Third International Conference on 3-D Digital Imaging and Modeling 145-152.

[21] Senin N, Colosimo BM, Pacella M (2013) Point set augmentation through fitting for enhanced ICP registration of point clouds in multisensor coordinate metrology. Robotics and Computer-Integrated Manufacturing 29: 39-52.

[22] Mukhopadhyay P, Chaudhuri B (2015) A survey of Hough Transform. Pattern Recognition 48: 993-1010.

[23] Guennebaud G, Germann M, Gross M (2008) Dynamic Sampling and Rendering of Algebraic Point Set Surfaces. Computer Graphics Forum: Proceedings of Eurographics.

[24] Haibin Z (2010) Multisensor integration and discrete geometry processing for coordinate metrology, PhD Thesis, ENS-Cachan, France.

[25] M. D. Carmo, Differential geometry of curves and surfaces, Prentice-Hall, Englewood Cliffs, New Jersey, 1976.

[26] Meyer M, Desbrun M, Schroder P, Barr AH (2002) Discrete differential-geometry perators for triangulated 2-manifolds. Proceedings of Visualization and Mathematics 35-57.

[27] Taubin G (1995) Estimating the tensor of curvature of a surface from a polyhedral 
approximation. Proceedings of IEEE Conference on Computer Vision and Pattern Recognition 902-907.

[28] Srinark T (2008) Lecture note on differential geometry.

[29] Cohen-Steiner D, Morvan JM (2003) Restricted Delaunay Triangulation and Normal Cycle. Proceedings of 19th Annual ACM Symposium on Computational Geometry 312321.

[30] Sapidis N, Besl P (1995) Direct construction of polynominal surfaces from dense range images through region growing, Transactions of ACM on Graphics 14: 171-2005.

[31] Chuang M, Luo L, Brown BJ, Rusinkiewicz S, Kazhdan M (2009) Estimating the Laplace-Beltrami operator by restricting functions. Proceedings of Eurographics Symposium on Geometry 28: 1475-1484.

[32] Koenderink JJ, Van Doorn RJ (1992) Surface shape and curvature scales. Journal of Imaging and Vision Computing 10: 557-565.

[33] Lomonosov E, Chetverikov D, Ekart A (2004) Fully automatic, robust and precise alignment of measured 3D surfaces for arbitrary orientations. Proceedings of the 28th Workshop of the Austrian Association for Pattern Recognition 39-46.

[34] Merlin PM, Farber DJ (1975) A parallel mechanism for detecting curves in pictures. IEEE Trans Comput 96-98.

[35] Ballard DH (1981) Generalizing the Hough Transform to detect arbitrary shapes. Pattern Recognit 13: 111-122.

[36] El-Hayeka N, Nouira H, Anwer N, Gibaru O, Damak M (2014) A new method for aspherical surface fittingwith large-volume datasets. Precision Engineering 38: 935947. 


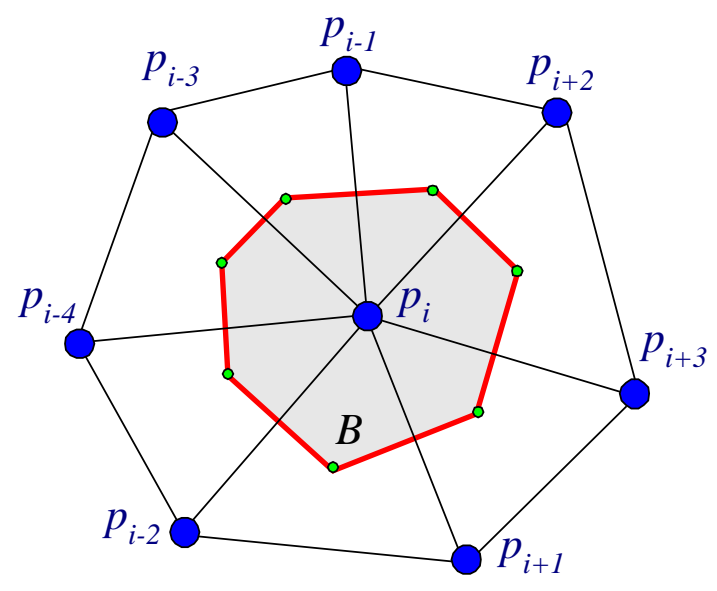

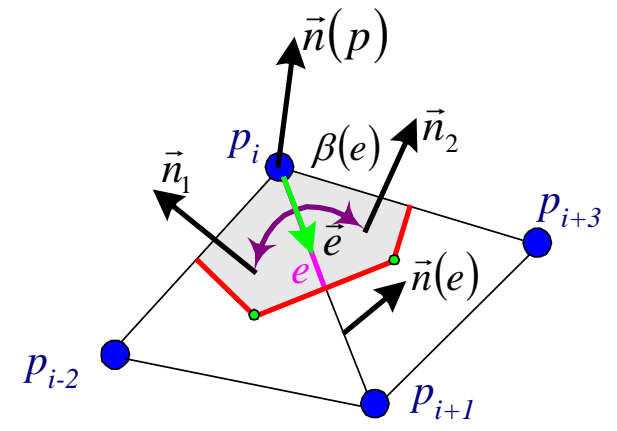

(b)

(a)

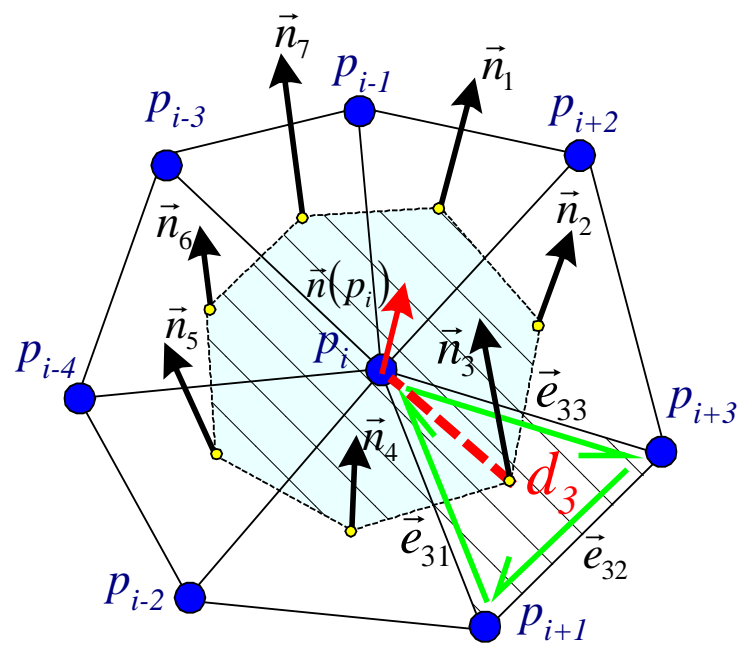

(c)

Fig.1. (a) Illustration of Voronoi-cell generation; (b) Illustration of the parameters used for $\boldsymbol{H}$ formulation; (c) Illustration of the surface normal estimation. 


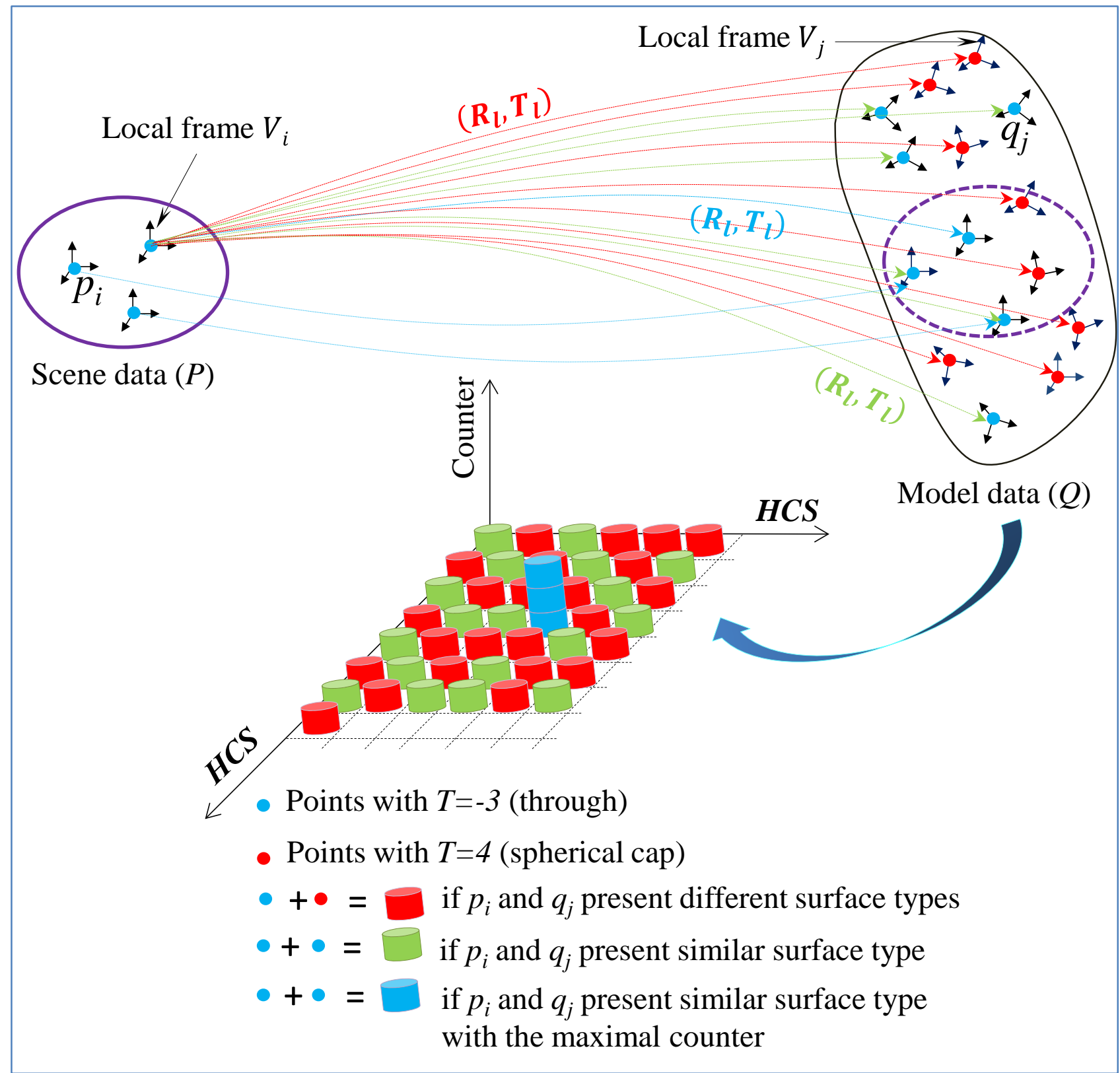

Fig.2. Schema of the basic Hough Transform (HT) method where the 6D HCS is represented by 2D space for the illustration. 


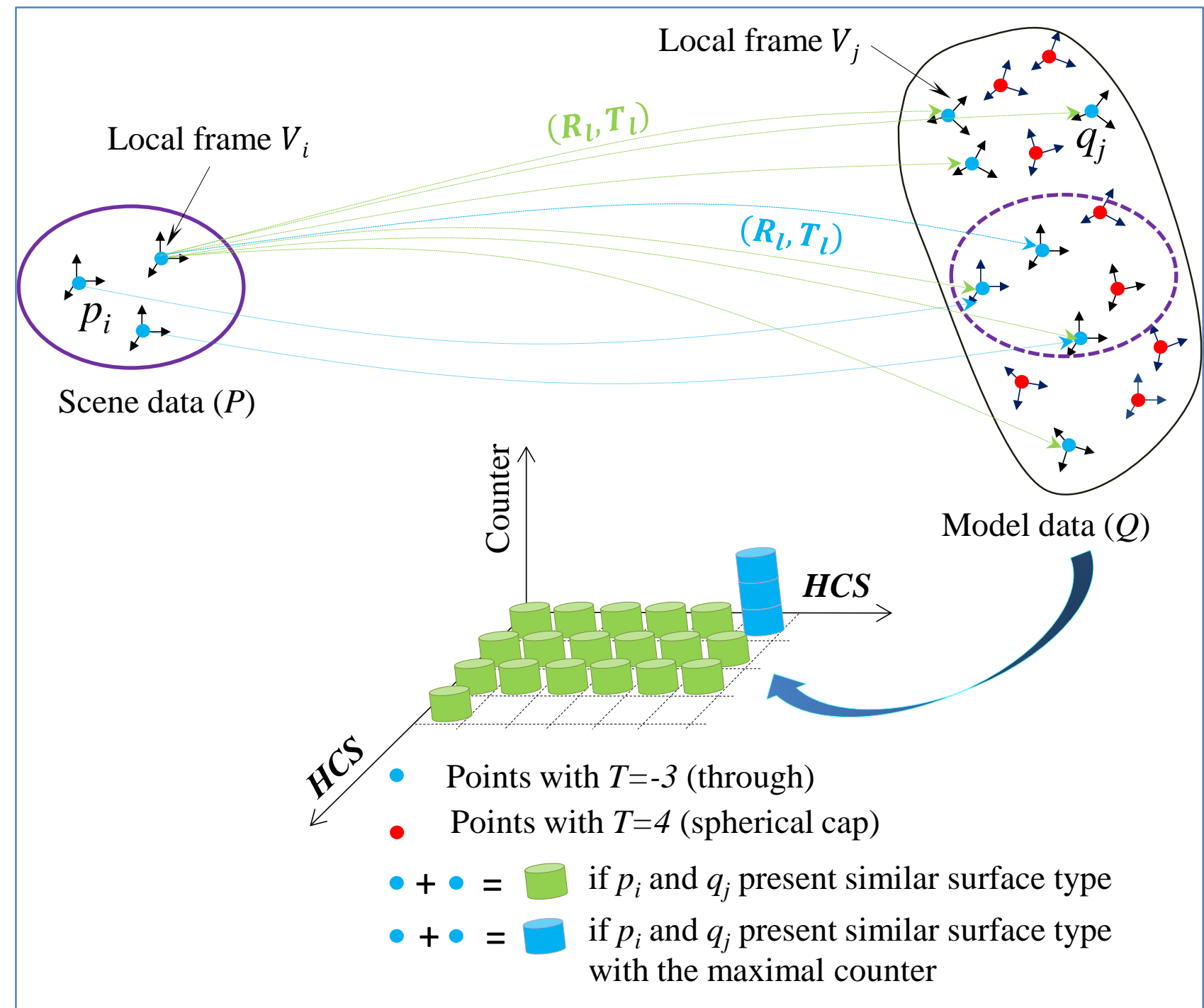

Fig.3. Schema of the improved Hough Transform (HT) method where the 6D HCS is represented by $2 \mathrm{D}$ space for the illustration. 


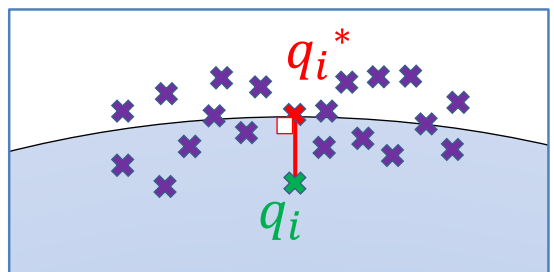

Fig.4. Local fitted algebraic sphere u(X). 


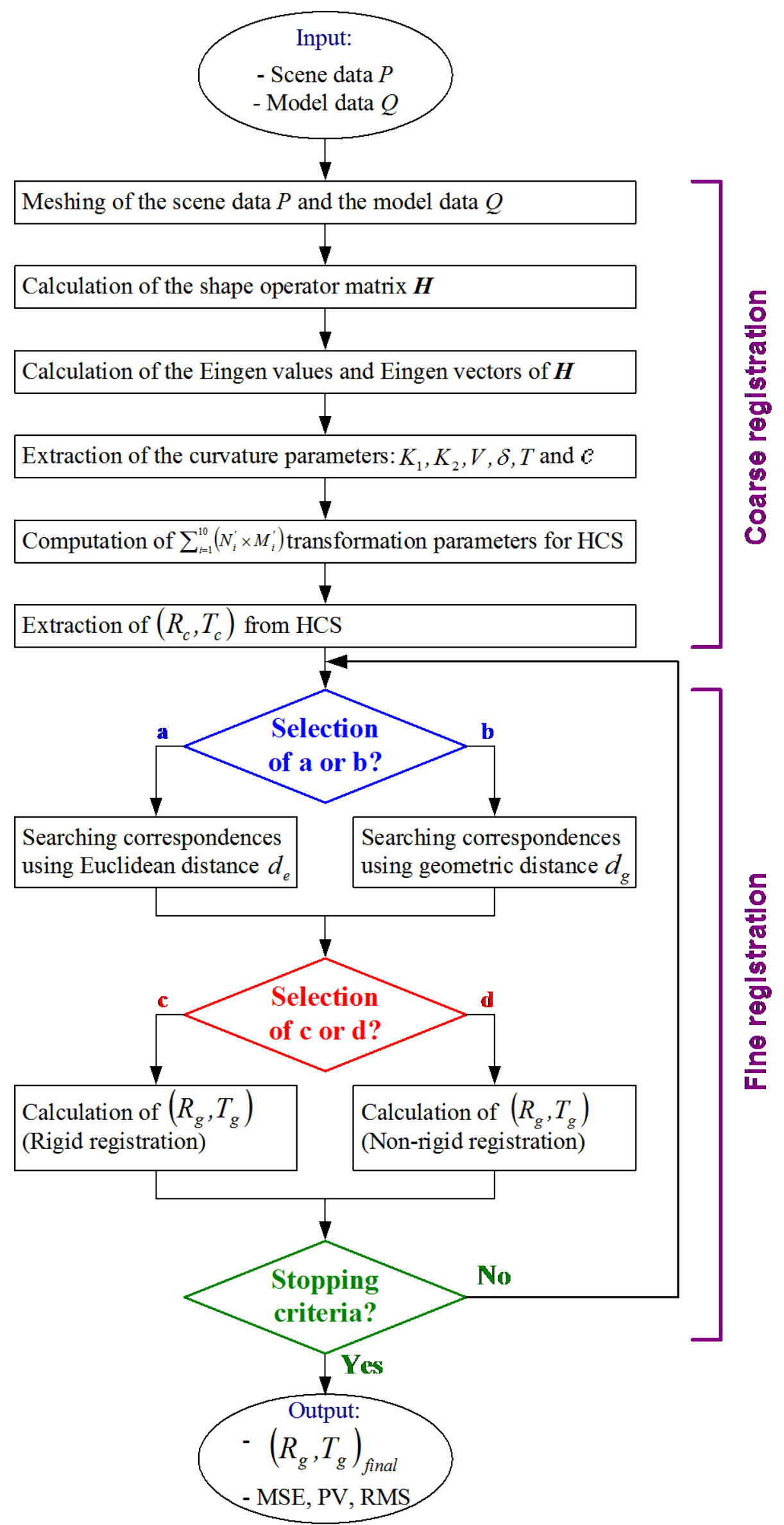

Fig.5. Sequential algorithm for the whole registration process. 


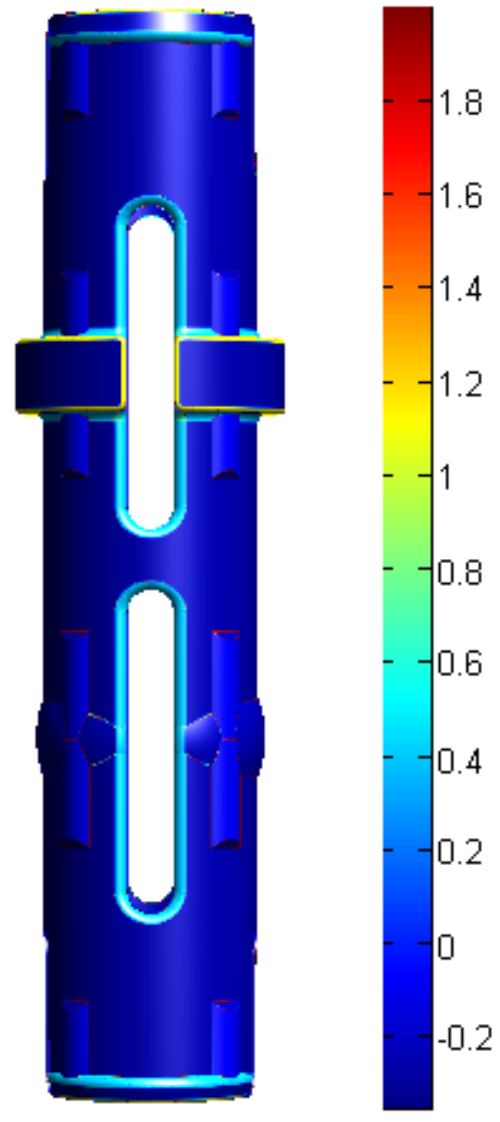

(a)

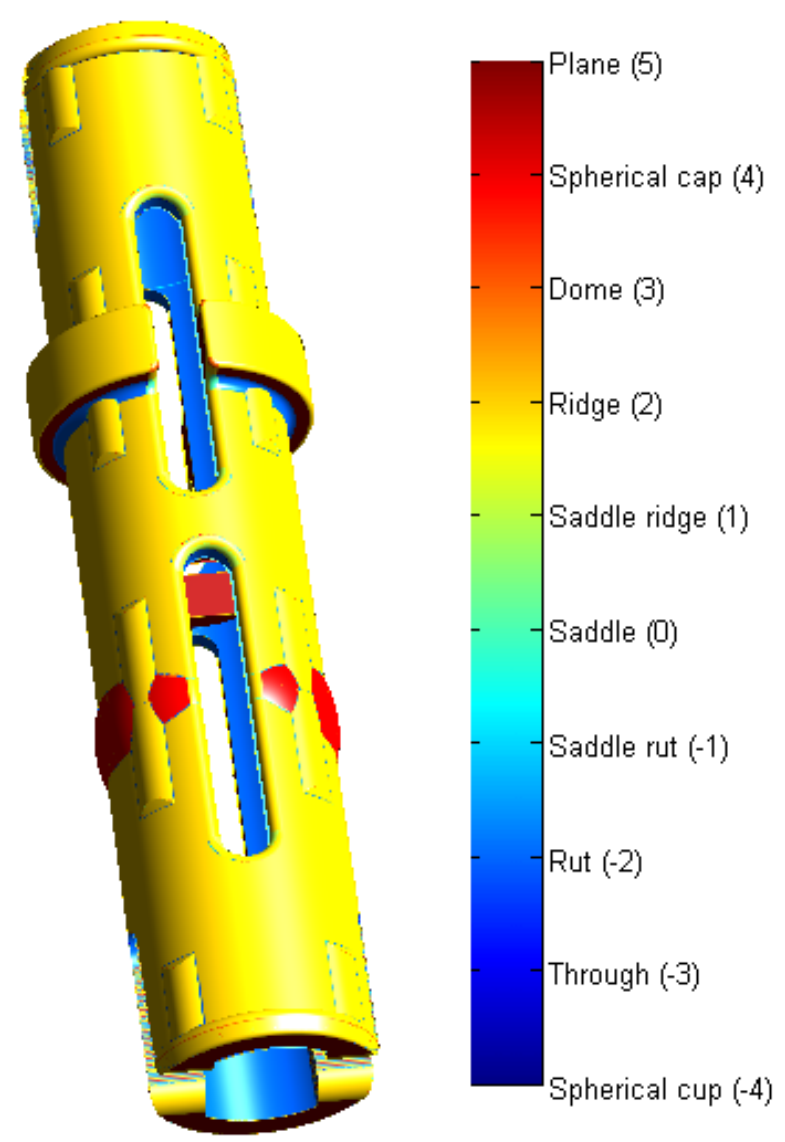

(b)

Fig.6. Curvature parameters maps of the LEGO connector: (a) Curvatness $\mathcal{C}$ map; (b) Surface type $T$ map. 

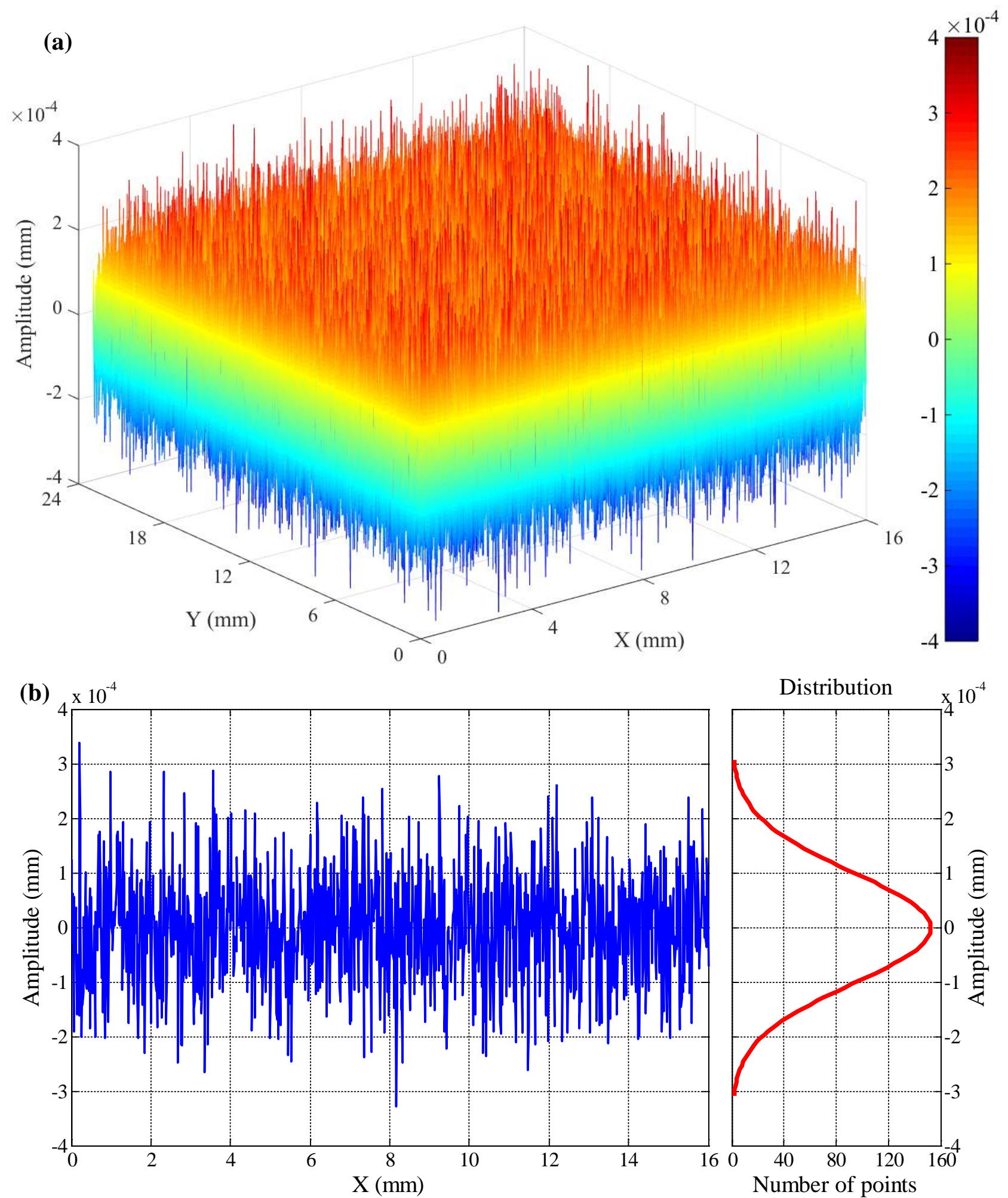

Fig.7. Simulated Gaussian noise ( $\mu=0, \sigma=8 \mathrm{~nm}$ ): (a) 3D plot; (b) 2D distribution at the middle section in the xz-plane. 


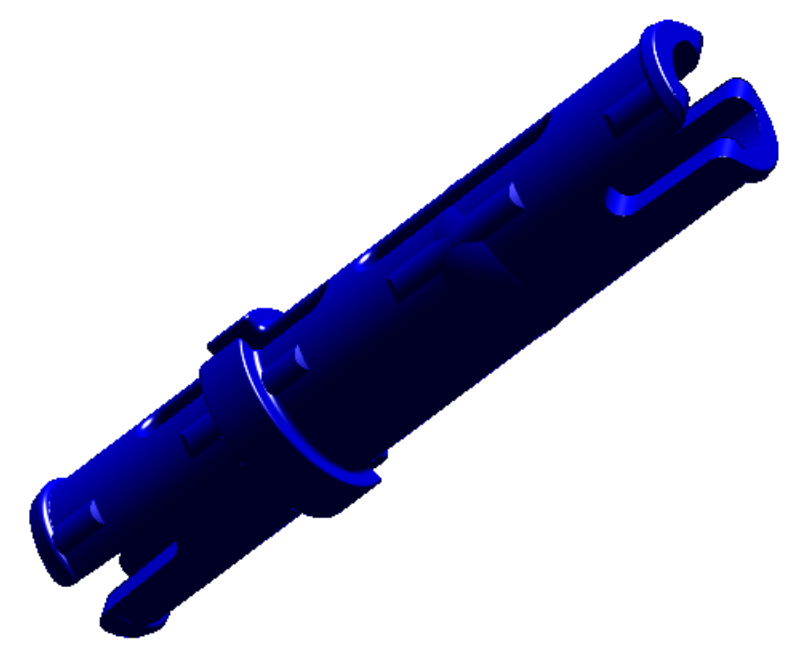

(a)

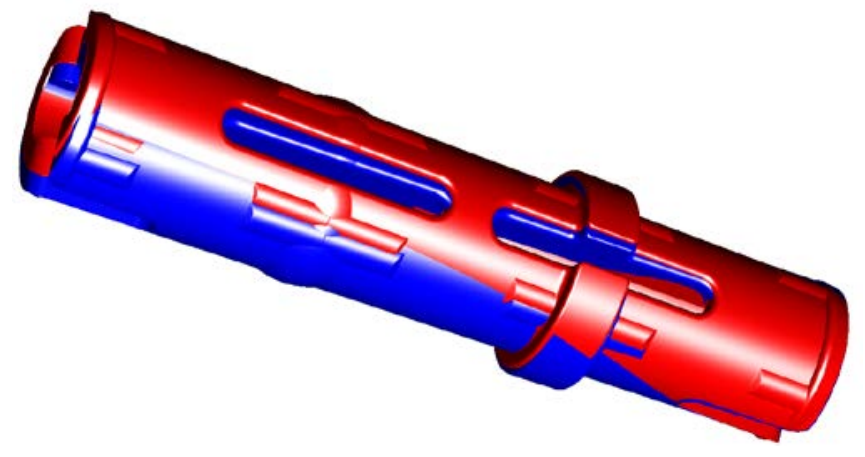

(b)

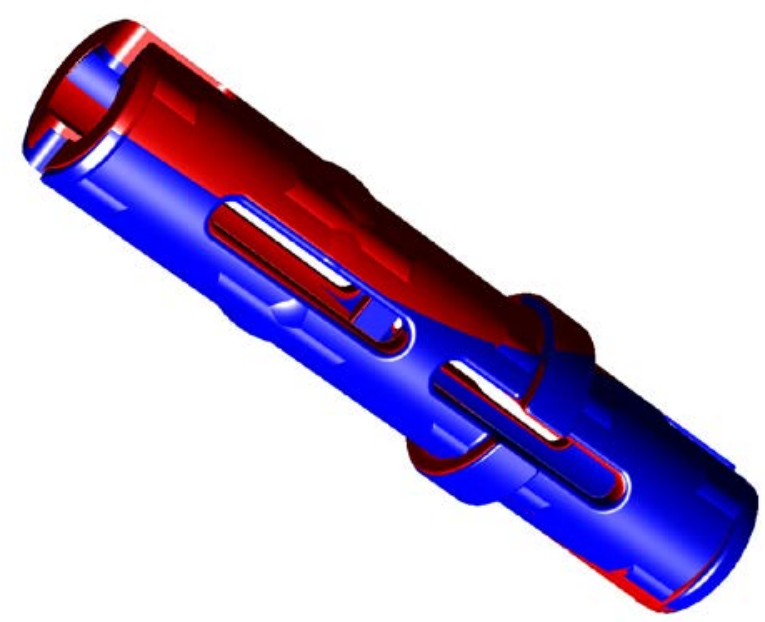

(c)

Fig.8. Study of the robustness of the developed three algorithms on simulated connector data: (a) Initial alignement (red: model data, blue: scene data), translations: $T_{x}=2 \mathrm{~mm}, T_{y}=-3$ and $T_{z}=-1$ $\mathrm{mm}$ ) and rotations: ( $R_{x}=-0.5 \mathrm{rad}, R_{y}=-0.01 \mathrm{rad}$ and $R_{z}=0.5 \mathrm{rad}$ ); (b) Coarse alignement; (c) Fine alignement. 


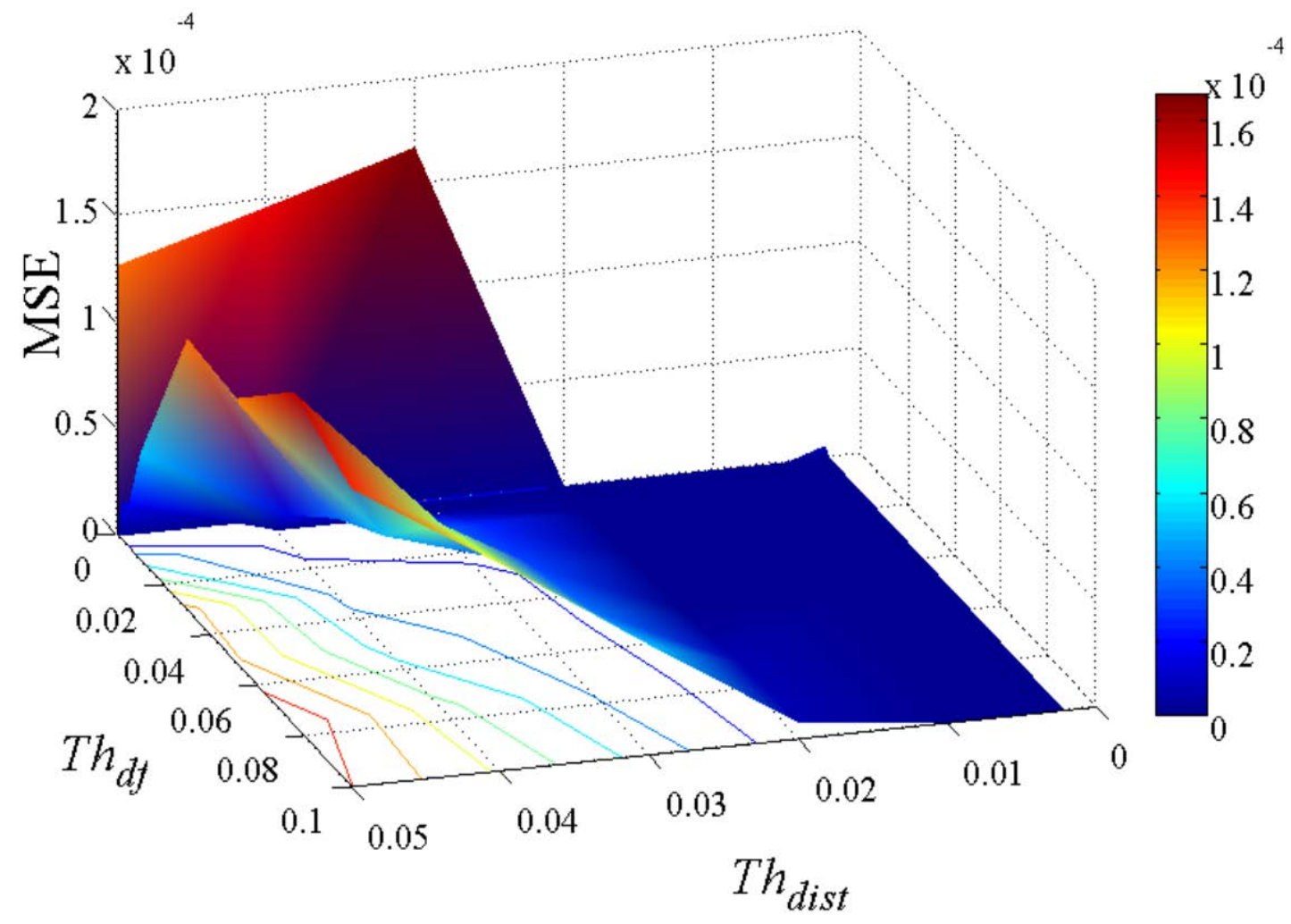

Fig.9. Evolution of the MSE versus the threshold of $d_{f}$ denoted as $T h_{d f}$ and the threshold of the Euludien distance $R_{p q}=\min _{q_{i} \in \psi\left(p_{i}\right)} \operatorname{dist}\left(p_{i}, q_{i}\right)$ denoted as $T h_{\text {dist }}$. 


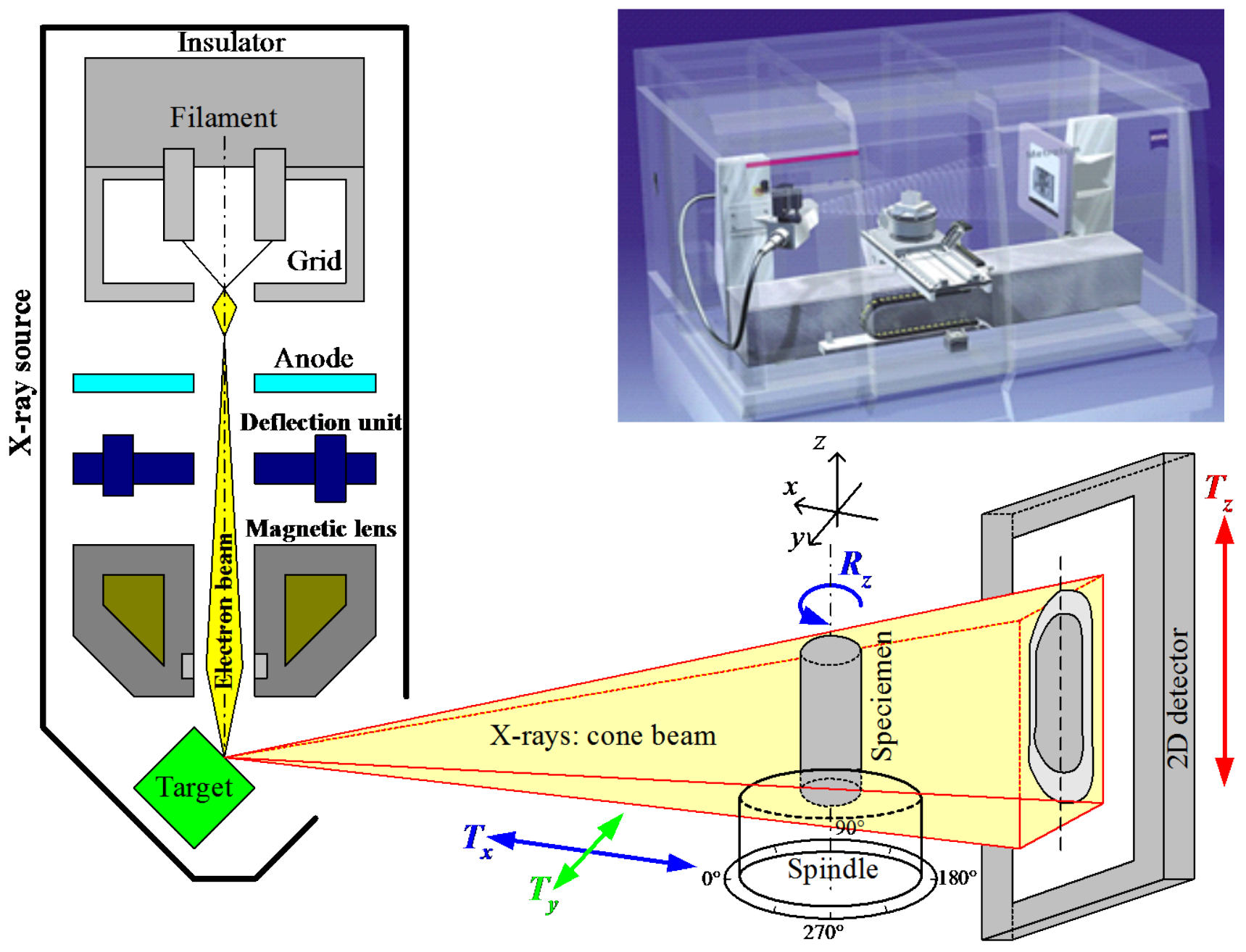

Fig.10. Schema of the $\mu \mathrm{CT}$ equipped with 2D flat panel detector with cone beam (Carl Zeiss METROTOM 800CT). 


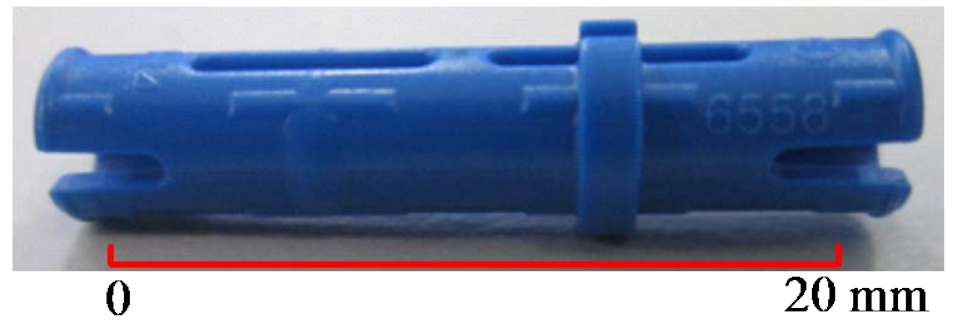

Fig.11. Photo of LEGO connector. 


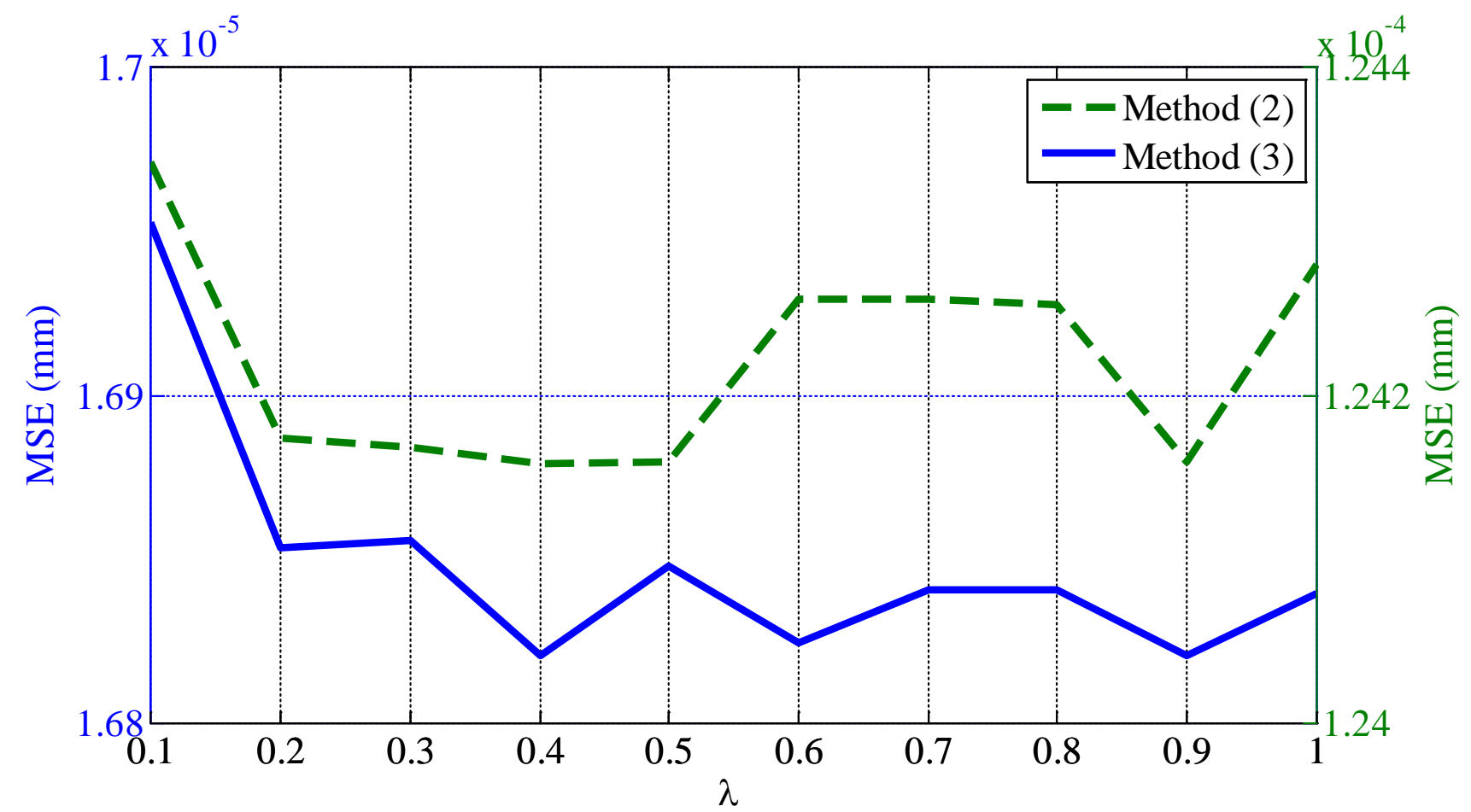

Fig.12. Influence of $\lambda$ parameters on MSE results related to the application on the $\mu C T$ measurement data. 

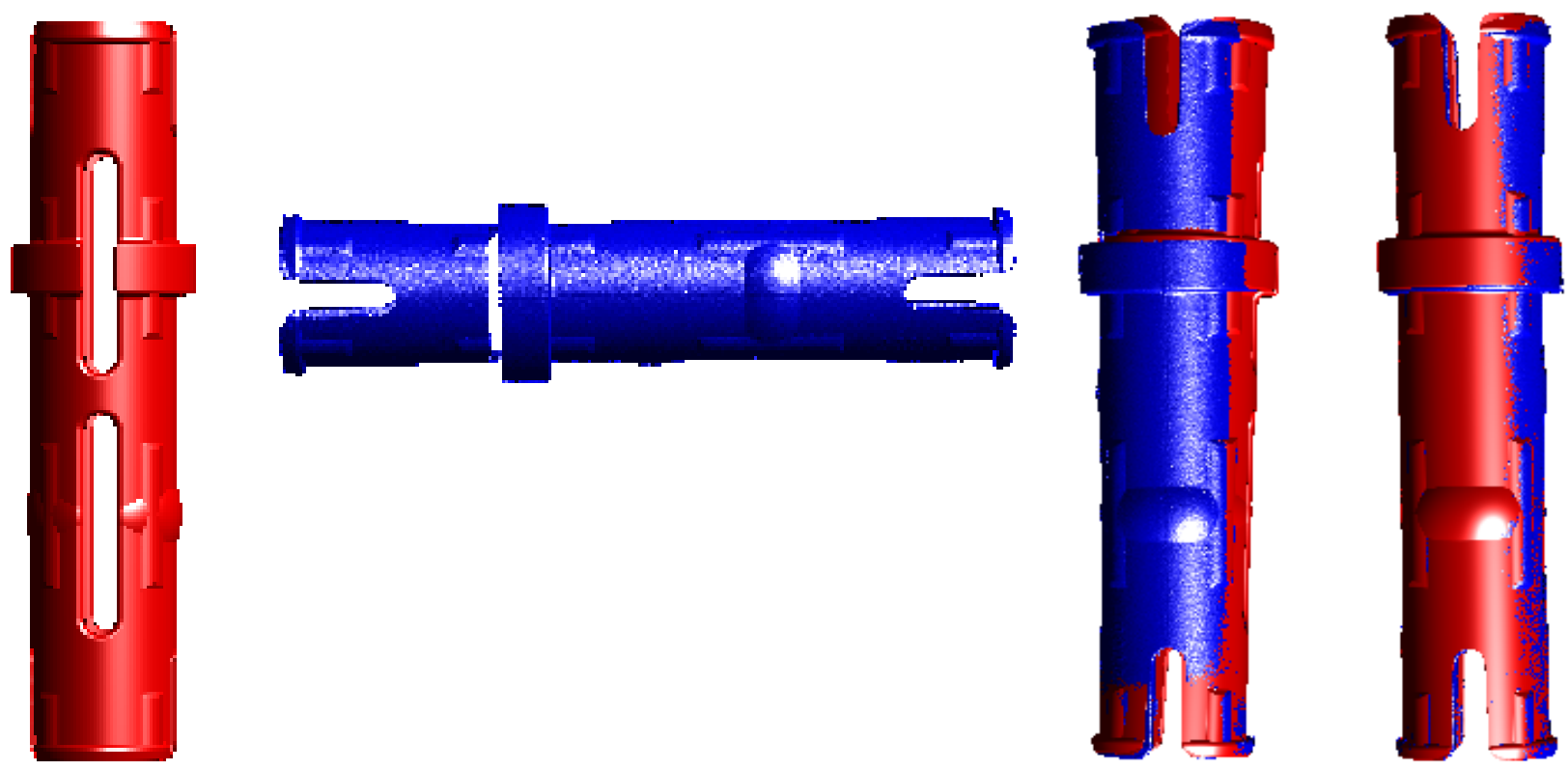

(a)

(b)

(c)

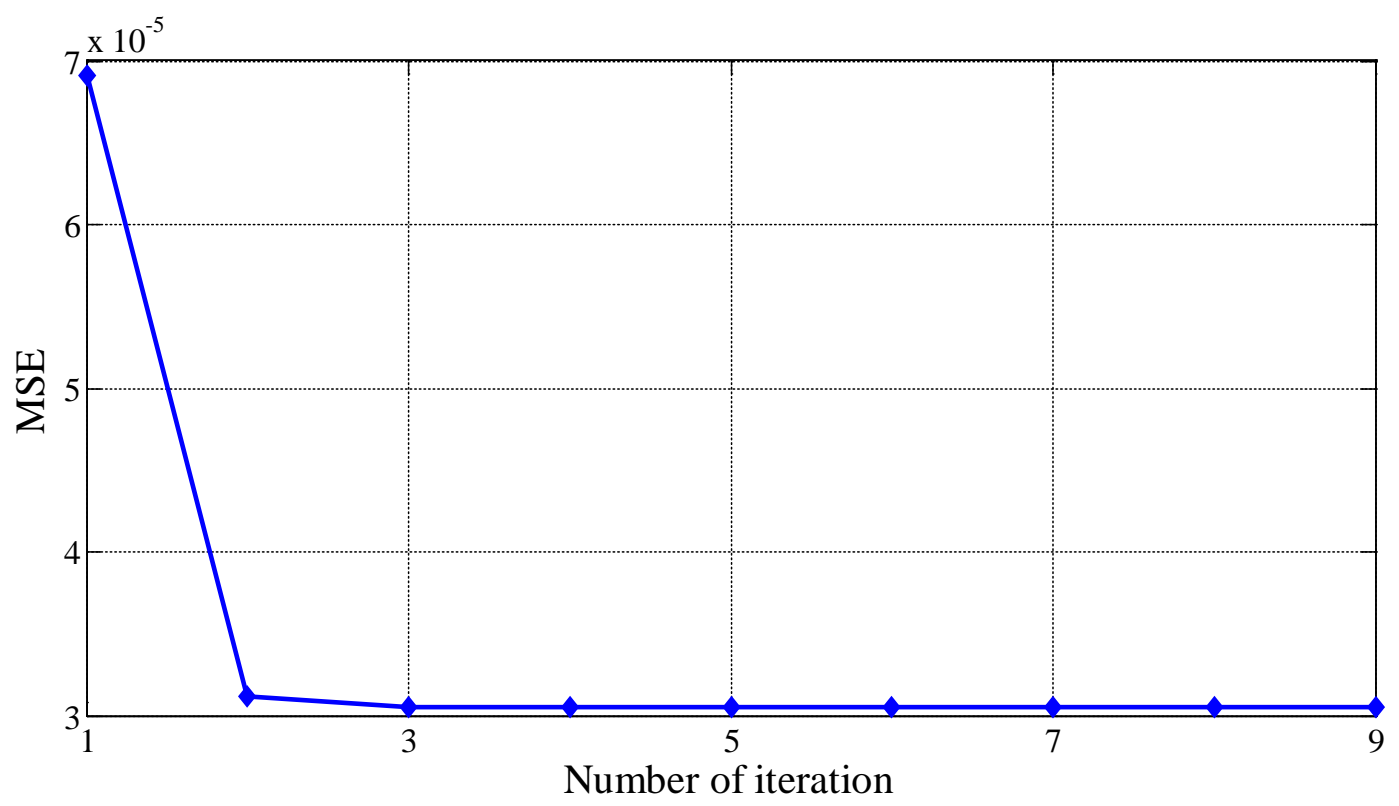

(d)

Fig.13. LEGO connector: registration of both CAD data and CT measurement data: (a) Intial alignement (red: CAD, blue: CT measurement); (b) Corse alignement; (c) Fine alignement; (d) Evolution of the MSE versus the number of iteration. 


\begin{tabular}{|c|c|c|c|}
\hline Surface type & Shape index interval & Type label & Standardized colour \\
\hline Spherical cup & $\delta \in\left[-1,-\frac{7}{8}\right]$ & $T=-4$ & \\
\hline Through & $\left.\delta \in]-\frac{7}{8},-\frac{5}{8}\right]$ & $T=-3$ & \\
\hline Rut & $\left.\delta \in]-\frac{5}{8},-\frac{3}{8}\right]$ & $T=-2$ & \\
\hline Saddle rut & $\left.\delta \in]-\frac{3}{8},-\frac{1}{8}\right]$ & $T=-1$ & \\
\hline Saddle & $\left.\delta \in]-\frac{1}{8}, \frac{1}{8}\right]$ & $T=0$ & \\
\hline Saddle ridge & $\left.\delta \in] \frac{1}{8}, \frac{3}{8}\right]$ & $T=1$ & \\
\hline Ridge & $\left.\delta \in] \frac{3}{8}, \frac{5}{8}\right]$ & $T=2$ & \\
\hline Dome & $\left.\delta \in] \frac{5}{8}, \frac{7}{8}\right]$ & $T=3$ & \\
\hline Spherical cap & $\left.\delta \in\rfloor \frac{7}{8}, 1\right]$ & $T=4$ & \\
\hline Plane & $\delta=2$ & $T=5$ & \\
\hline
\end{tabular}

Tab.1. Surface types specified by shape index intervals. 


\begin{tabular}{lcc}
\hline \hline \multicolumn{1}{c}{ Surface type } & Parameter $\boldsymbol{\alpha}_{\boldsymbol{i}}$ & Parameter $\boldsymbol{\beta}_{\boldsymbol{i}}$ \\
\hline Spherical cup ( $\mathrm{T}=-4)$ & 0.9 & 0.1 \\
Through ( $\mathrm{T}=-3)$ & 0.8 & 0.2 \\
Rut ( $\mathrm{T}=-2)$ & 0.7 & 0.3 \\
Saddle rut (T=-1) & 0.6 & 0.4 \\
Saddle ( $\mathrm{T}=0)$ & 0.5 & 0.5 \\
Saddle ridge ( $\mathrm{T}=1)$ & 0.4 & 0.6 \\
Ridge (T=2) & 0.3 & 0.7 \\
Dome (T=3) & 0.2 & 0.8 \\
Spherical cap (T=4) & 0.1 & 0.9 \\
Plane (T=5) & 0 & 1
\end{tabular}

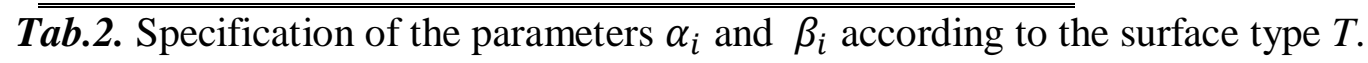




\begin{tabular}{cccccccc}
\hline \hline Method & $\begin{array}{c}\text { Gen. } P V \\
(\mathrm{~nm})\end{array}$ & $\begin{array}{c}\text { Est. } P V \\
(\mathrm{~nm})\end{array}$ & $\begin{array}{c}|\Delta(P V)| \\
(\mathrm{nm})\end{array}$ & $\begin{array}{c}\text { Gen. } R M S \\
(\mathrm{~nm})\end{array}$ & $\begin{array}{c}\text { Est. } R M S \\
(\mathrm{~nm})\end{array}$ & $\begin{array}{c}|\Delta(R M S)| \\
(\mathrm{nm})\end{array}$ & MSE \\
\hline \multirow{3}{*}{$(1)$} & $1.99510^{-5}$ & $1.99510^{-5}$ & $2.31410^{-15}$ & $4.65010^{-6}$ & $4.65010^{-6}$ & $9.84810^{-17}$ & $7.75110^{-31}$ \\
\cline { 2 - 8 } & $2.08410^{-4}$ & $2.08410^{-4}$ & $1.15910^{-15}$ & $4.41710^{-5}$ & $4.41710^{-5}$ & $3.59010^{-17}$ & $7.96210^{-31}$ \\
\cline { 2 - 8 } & $4.17910^{-4}$ & $4.11910^{-4}$ & $5.63210^{-8}$ & $9.19510^{-5}$ & $9.20010^{-5}$ & $5.63210^{-8}$ & $6.09910^{-11}$ \\
\hline \multirow{3}{*}{$(2)$} & $1.9410^{-5}$ & $6.10510^{-5}$ & $4.11010^{-5}$ & $4.6410^{-6}$ & $1.43310^{-4}$ & $1.38710^{-4}$ & $1.11510^{-4}$ \\
\hline & $2.0510^{-4}$ & $1.37510^{-4}$ & $7.08610^{-5}$ & $4.4110^{-5}$ & $1.49910^{-4}$ & $1.05610^{-4}$ & $3.08110^{-6}$ \\
\hline \multirow{3}{*}{$(3)$} & $4.1510^{-4}$ & $3.60810^{-4}$ & $5.70510^{-5}$ & $9.1910^{-5}$ & $1.70210^{-4}$ & $7.82410^{-5}$ & $3.08310^{-6}$ \\
\hline & $1.99510^{-5}$ & $1.91910^{-5}$ & $7.60110^{-7}$ & $4.65010^{-6}$ & $4.65110^{-6}$ & $8.83610^{-10}$ & $2.02510^{-11}$ \\
\hline & $2.08410^{-4}$ & $2.05510^{-4}$ & $2.44610^{-6}$ & $4.41710^{-5}$ & $4.41310^{-5}$ & $2.71510^{-8}$ & $1.84210^{-9}$ \\
\hline & $4.17910^{-4}$ & $4.15910^{-4}$ & $1.99610^{-6}$ & $9.19510^{-5}$ & $9.19510^{-5}$ & $1.71910^{-9}$ & $8.10610^{-9}$ \\
\hline
\end{tabular}

Tab.3. Results related to the first simulation (Gen.: Generated, Est.: Estimated). 


\begin{tabular}{|c|c|c|c|c|c|c|c|}
\hline Method & $\begin{array}{c}\text { Gen. } P V \\
(\mathrm{~nm})\end{array}$ & $\begin{array}{l}\text { Est. } P V \\
(\mathrm{~nm})\end{array}$ & $\begin{array}{c}|\Delta(P V)| \\
(\mathrm{nm})\end{array}$ & $\begin{array}{c}\text { Gen. } R M S \\
(\mathrm{~nm})\end{array}$ & $\begin{array}{c}\text { Est. } R M S \\
(\mathrm{~nm})\end{array}$ & $\begin{array}{c}|\Delta(R M S)| \\
(\mathrm{nm})\end{array}$ & MSE \\
\hline \multirow{2}{*}{ (1) } & $1.99510^{-5}$ & - & \multirow{2}{*}{$6.46510^{-8}$} & $4.65010^{-6}$ & - & \multirow{2}{*}{$3.84110^{-7}$} & \multirow{2}{*}{$7.39010^{-31}$} \\
\hline & $4.17910^{-4}$ & $4.17910^{-4}$ & & $9.19510^{-5}$ & $9.23310^{-5}$ & & \\
\hline \multirow{2}{*}{ (2) } & $1.99510^{-5}$ & - & \multirow{2}{*}{$1.61810^{-4}$} & $4.65010^{-6}$ & - & \multirow{2}{*}{$1.91110^{-4}$} & \multirow{2}{*}{$2.32010^{-8}$} \\
\hline & $4.17910^{-4}$ & $5.79810^{-4}$ & & $9.1910^{-5}$ & $2.83010^{-4}$ & & \\
\hline \multirow{2}{*}{ (3) } & $1.99510^{-5}$ & - & \multirow{2}{*}{$3.00210^{-6}$} & $4.65010^{-6}$ & - & \multirow{2}{*}{$4.30710^{-7}$} & \multirow{2}{*}{$8.17010^{-9}$} \\
\hline & $4.17910^{-4}$ & $4.14910^{-4}$ & & $9.19510^{-5}$ & $9.23810^{-5}$ & & \\
\hline
\end{tabular}

Tab.4. Results related to the second simulation. Gaussian noise amplitude of $20 \mathrm{~nm}$ is added to model data $Q$, while Gaussian noise amplitude of $400 \mathrm{~nm}$ is added to scene data $P$. 


\begin{tabular}{|c|c|c|c|c|c|c|c|}
\hline Method & $\begin{array}{c}\text { Gen. } P V \\
\text { (nm) }\end{array}$ & $\begin{array}{l}\text { Est. } P V \\
(\mathrm{~nm})\end{array}$ & $\begin{array}{c}|\Delta(P V)| \\
(\mathrm{nm})\end{array}$ & $\begin{array}{c}\text { Gen. } R M S \\
(\mathrm{~nm})\end{array}$ & $\begin{array}{c}\text { Est. } R M S \\
(\mathrm{~nm})\end{array}$ & $\begin{array}{c}|\Delta(R M S)| \\
(\mathrm{nm})\end{array}$ & MSE \\
\hline \multirow{2}{*}{ (1) } & $4.17910^{-4}$ & - & \multirow{2}{*}{$6.46510^{-8}$} & $9.19510^{-5}$ & - & \multirow{2}{*}{$3.84110^{-7}$} & \multirow{2}{*}{$7.39010^{-31}$} \\
\hline & $1.99510^{-5}$ & $4.17810^{-4}$ & & $4.65010^{-6}$ & $9.23910^{-5}$ & & \\
\hline \multirow{2}{*}{ (2) } & $4.17910^{-4}$ & - & \multirow{2}{*}{$1.34210^{-4}$} & $9.19510^{-5}$ & - & \multirow{2}{*}{$1.13310^{-4}$} & \multirow{2}{*}{$2.47010^{-8}$} \\
\hline & $1.99510^{-5}$ & $4.31610^{-4}$ & & $4.65010^{-6}$ & $2.05210^{-4}$ & & \\
\hline \multirow{2}{*}{ (3) } & $4.17910^{-4}$ & - & \multirow{2}{*}{$3.33710^{-6}$} & $9.19510^{-5}$ & - & \multirow{2}{*}{$4.41410^{-7}$} & \multirow{2}{*}{$8.18010^{-9}$} \\
\hline & $1.99510^{-5}$ & $4.14510^{-4}$ & & $4.65010^{-6}$ & $9.23910^{-5}$ & & \\
\hline
\end{tabular}

Tab.5. Results related to the second simulation. Gaussian noise amplitude of $400 \mathrm{~nm}$ is added to model data $Q$, while Gaussian noise amplitude of $20 \mathrm{~nm}$ is added to scene data $P$. 


\section{Performances features}

\begin{tabular}{ll}
\hline Tube & $130 \mathrm{kV} / 39 \mathrm{~W}$ \\
2D detector & $1900 \times 1512$ pixels \\
Measuring range & $\varnothing 125 \times 150 \mathrm{~mm}$ \\
Lifting table & $290 \mathrm{~mm}$ \\
Source-detector distance & $800 \mathrm{~mm}$ \\
\hline \hline
\end{tabular}

Tab.6. Carl Zeiss METROTOM 800 specifications. 


\begin{tabular}{lccc}
\hline \hline Method & $P V(\mathrm{~mm})$ & $R M S(\mathrm{~mm})$ & MSE \\
\hline$(1)$ & $8.795110^{-2}$ & $4.17810^{-3}$ & $3.05010^{-5}$ \\
\hline$(2)$ & $8.784610^{-2}$ & $4.19710^{-3}$ & $1.24110^{-4}$ \\
\hline$(3)$ & $8.802710^{-2}$ & $4.17310^{-3}$ & $1.68210^{-5}$ \\
\hline \hline
\end{tabular}

Tab.7. Results related to the CAD and the $\mu \mathrm{CT}$ measurement alignement. 